\title{
INDEPENDENT INTEGRAL BASES AND A CHARAC- TERIZATION OF REGULAR SURFACES
}

\author{
BY \\ H. T. MUHLY(1)
}

Introduction. The fundamental paper Theorie der algebraischen Funktionen einer Veränderlichen of Dedekind and Weber opened a wide field of research. The methods which these authors brought into play in the study of algebraic functions of one variable have lent themselves readily, from the conceptual point of view at least, to generalization to the case of several variables. However, even if one restricts himself to the case of algebraic functions of two variables he finds a sharp line of demarcation in the analogy with the one variable case when he attacks problems of enumeration such as the problem of Riemann-Roch.

Several years ago Zariski made the conjecture that the analogy between the theory of algebraic curves and the theory of algebraic surfaces would extend much further in the case of regular surfaces, that is surfaces whose arithmetic and geometric genera coincide. The objective of this paper is to establish the truth of this conjecture and to show that this fact is in a sense characteristic of regular surfaces.

A tool which proved to be most useful to Dedekind and Weber in deriving the Riemann-Roch theorem as well as other allied results was furnished by their so-called normal bases, the construction of which we shall describe briefly below.

Let $\Sigma$ be a field of algebraic functions of one variable $x$ c.ver a field of constants $K$, which is assumed to be maximally algebraic in $\Sigma$ and of characteristic zero. The field $\Sigma$ is then an algebraic extension of $K(x)$ of finite relative degree $\nu$. Let $\boldsymbol{D}$ denote the ring of integral functions of $x$ in $\Sigma$ and $\mathfrak{o}^{\prime}$ the ring of integral functions of $1 / x$ in $\Sigma$. If $\omega$ is an element of 0 , the "exponent" of $\omega$ (in symbols exp $\omega$ ) is defined to be the smallest integer $r$ such that $\omega / x^{r}$ is an element of $\mathfrak{D}^{\prime}$ (that is $\omega / x^{r} \subset \mathfrak{o}^{\prime}, \omega / x^{r-1} \mp \mathfrak{o}^{\prime}$ ). A set of functions $\lambda_{1}, \lambda_{2}, \cdots, \lambda_{v}$ is constructed as follows. Let $\lambda_{1}=1$, and let $\lambda_{2}$ be an element in $\mathfrak{D}$ of lowest exponent $r_{2}$, such that $\lambda_{2}$ does not satisfy a congruence of the form $\lambda_{2} \equiv c \lambda_{1}(\mathfrak{D} x)$ where $c \in K$. If $\lambda_{1}, \lambda_{2}, \cdots, \lambda_{i-1}$ have been selected, take $\lambda_{i}$ to be an element in $\boldsymbol{D}$ of lowest exponent which does not satisfy a congruence of the form $\lambda_{i} \equiv c_{1} \lambda_{1}+\cdots+c_{i-1} \lambda_{i-1}(\mathrm{D} x), c_{j} \in K, j=1, \cdots, i-1$. Dedekind and Weber show that this construction leads to a set of $\nu(=[\Sigma: K(x)])$ functions $\lambda_{1}, \lambda_{2}, \cdots, \lambda_{\nu}$ in $\mathbb{D}$ which have the following properties:

Presented to the Society, September 13, 1943; received by the editors September 10, 1942 and, in revised form, February 17, 1943.

(1) National Research Fellow, 1940-1941. 
(a) They are linearly independent over $K(x)$ and therefore form a modular basis for $\Sigma$ over $K(x)$.

(b) They form a modular basis for $\mathfrak{D}$ over the polynomial ring $K[x]$.

(c) If $\exp \lambda_{i}=r_{i}$, then $r_{1}(=0), 1 \leqq r_{2} \leqq \cdots \leqq r_{\nu}$. If $\omega$ is in $\mathfrak{D}$, and if $r_{h}$ is the last integer in the sequence $r_{1}, r_{2}, \cdots, r_{\nu}$ which is less than or equal to $\exp \omega$, then $\omega$ is of the form $\omega=P_{1}(x) \lambda_{1}+\cdots+P_{h}(x) \lambda_{h}$, and the degree of $P_{i}(x)$ (a polynomial) is less than or equal to $\left(\exp \omega-r_{i}\right)$.

(d) The quantities $\lambda_{i}^{\prime}=\lambda_{i} / x^{r_{i}}, i=1,2, \cdots, \nu$, form a base for $\mathfrak{o}^{\prime}$ over $K[1 / x]$ with the same properties relative to $D^{\prime}$ as the quantities $\lambda_{1}, \cdots, \lambda_{\nu}$ have relative to $\mathrm{D}$.

(e) $\sum_{i=2}^{v}\left(r_{i}-1\right)=p$, the genus of $\Sigma$.

Dedekind and Weber refer to such a set of functions as a normal base for 0 over $K[x]$.

When we pass to the case of several variables, $x_{1}, x_{2}, \cdots, x_{r}$, and let $\Sigma$ denote an algebraic extension of $K\left(x_{1}, x_{2}, \cdots, x_{r}\right)$, we must consider together with the integral closure $\mathrm{D}_{0}$ of the polynomial ring $K\left[x_{1}, x_{2}, \cdots, x_{r}\right]$ the integral closures $D_{i}$ of the polynomial rings $K\left[x_{1} / x_{i}, \cdots, x_{i-1} / x_{i}, 1 / x_{i}, x_{i+1} / x_{i}\right.$, $\left.\cdots, x_{r} / x_{i}\right], i=1,2, \cdots, r$, if we want to generalize the concepts mentioned above. It will be pointed out in $\$ 2$ that it is possible to define an integer, $\exp \omega$, for every element $\omega$ in $\mathfrak{o}_{0}$ (or in $\boldsymbol{D}_{i}$ ) with the property that if $\exp \omega=h$ then $\omega / x_{i}^{h} \subset \mathrm{o}_{i}, \omega / x_{i}^{h-1} \subset_{0_{i}}, i=1,2, \cdots, r$. A set of $\nu\left(=\left[\Sigma: K\left(x_{1}, x_{2}, \cdots, x_{r}\right)\right]\right)$ elements, $\lambda_{1}, \lambda_{2}, \cdots, \lambda_{\nu}$ in $D_{0}$ which form a modular base for $\mathfrak{D}_{0}$ over $K\left[x_{1}, x_{2}, \cdots, x_{r}\right]$ (and consequently form a base for $\Sigma$ over $\left.K\left(x_{1}, x_{2}, \cdots, x_{r}\right)\right)$ will be referred to as a $D W$-base for $0_{0}$ relative to $K\left[x_{1}, x_{2}, \cdots, x_{r}\right]$ if in addition it satisfies the following conditions.

(a) If $\exp \lambda_{i}=r_{i}$ then $r_{1}(=0), 1 \leqq r_{2} \leqq r_{3} \leqq \cdots \leqq r_{\nu}$, and if $\omega$ is in $D_{0}$ and $r_{h}$ is the last integer.in the sequence $r_{1} \leqq r_{2} \leqq \cdots \leqq r_{\nu}$ which is less than or equal to $\exp \omega$, then $\omega$ is of the form $\omega=P_{1}(x) \lambda_{1}+P_{2}(x) \lambda_{2}+\cdots+P_{h}(x) \lambda_{h}$ where the polynomial $P_{i}$ is of degree less than or equal to $\left(\exp \omega-r_{i}\right)$.

(b) The quantities $\lambda_{i}^{(j)}=\lambda_{i} / x_{j}^{r_{i}}, j=1,2, \cdots, r ; i=1,2, \cdots, \nu$ have the same properties relative to the ring $o_{j}$ as the elements $\lambda_{1}, \lambda_{2}, \cdots, \lambda_{v}$ have relative to $\mathrm{D}_{0}$.

In $\S 2$ we show that if $\xi_{0}^{*}, \xi_{1}^{*}, \cdots, \xi_{n}^{*}$ are the homogeneous coordinates of the general point of an arithmetically normal variety $V_{r}$ in $P_{n}$ and if every element of the integrally closed ring $0^{*}=K\left[\xi_{0}^{*}, \xi_{1}^{*}, \cdots, \xi_{n}^{*}\right]$ depends integrally on the algebraically independent elements $\xi_{0}^{*}, \xi_{1}^{*}, \cdots, \xi_{r}^{*}$ then the existence of a DW-base in $0_{0}=K\left[\xi_{1}^{*} / \xi_{0}^{*}, \xi_{2}^{*} / \xi_{0}^{*}, \cdots, \xi_{n}^{*} / \xi_{0}^{*}\right]$ relative to the polynomial ring $K\left[\xi_{1}^{*} / \xi_{0}^{*}, \xi_{2}^{*} / \xi_{0}^{*}, \cdots, \xi_{r}^{*} / \xi_{0}^{*}\right]$ is equivalent to the existence of a modular base for $0^{*}$ over $K\left[\xi_{0}^{*}, \xi_{1}^{*}, \cdots, \xi_{r}^{*}\right]$ consisting of linearly independent homogeneous elements.

It is well known that such modular bases do not exist in general in rings such as $0^{*}$, so that DW-bases likewise do not always exist.

In this paper we shall prove that if $\xi_{0}^{*}, \xi_{1}^{*}, \cdots, \xi_{n}^{*}$ are homogeneous 
coordinates along a nonsingular, normal model of a field $\Sigma$ of degree of transcendency two relative to $K$, then the existence of a modular base for $0^{*}$ over $K\left[\xi_{0}^{*}, \xi_{1}^{*}, \xi_{2}^{*}\right]$ consisting of $\nu\left(=\left[\Sigma: K\left(\xi_{1}^{*} / \xi_{0}^{*}, \xi_{2}^{*} / \xi_{1}^{*}\right)\right]\right)$ linearly independent homogeneous elements implies that the field $\Sigma$ is regular. Moreover, if $F$ is a nonsingular model of a regular field $\Sigma$ and if $\mathrm{o}_{h}^{*}$ is the ring of homogeneous coordinates along a derived normal surface $F^{(h)}$ of $F$ belonging to a sufficiently high character of homogeneity $h[9]\left({ }^{2}\right)$, then $\mathbf{b}_{h}{ }^{*}$ has an independent homogeneous modular base relative to a suitably selected set of independent variables.

The proof of the first part of this statement, to the effect that the existence of a base implies regularity, is completely arithmetic in nature. For the proof of the converse, however, we found it necessary to use a lemma of Severi [6] which this author uses in his derivation of the Riemann-Roch theorem for surfaces. The proof of the converse by means of totally arithmetic arguments presents auxiliary problems requiring considerable investigation in themselves. We have not as yet been able to cope with all of the difficulties arising in connection with them.

Throughout this paper we shall use the term normal to mean arithmetically normal. The coefficient field $K$ is always assumed to be algebraically closed and of characteristic zero.

1. Definitions and preliminary remarks. Let $\Sigma$ be a field of algebraic functions of degree of transcendency $r$ relative to a subfield $K$. By a prime divisor of $\Sigma$ we shall mean any $(r-1)$-dimensional valuation of $\Sigma$. If $\Im$ is any integral domain in $\Sigma$, and if $\mathfrak{B}$ is a prime divisor whose $v$-ring $\mathfrak{B}(\mathfrak{B})$ contains $\Im$, then $\mathfrak{B}$ is said to be of first or second kind with respect to $\Im$ according as the center $\left(^{3}\right)$ of $\mathfrak{B}$ in $\Im$ is or is not $(r-1)$-dimensional.

If $\xi_{0}^{*}, \xi_{1}^{*}, \cdots, \xi_{n}^{*}$ are the homogeneous coordinates of the general point of a projective model $V_{r}$ of $\Sigma$, and if $0^{*}=K\left[\xi_{0}^{*}, \xi_{1}^{*}, \cdots, \xi_{n}^{*}\right]$ is the associated integral domain, then according to a well known theorem of Noether, a nonsingular projective transformation can be found which will insure that the first $r+1 \xi^{*}$ 's, $\xi_{0}^{*}, \xi_{1}^{*}, \cdots, \xi_{r}^{*}$ are algebraically independent and that the remaining ones, $\xi_{r+1}^{*}, \cdots, \xi_{n}^{*}$ (and hence the entire ring $0^{*}$ ), will be integrally dependent on $\xi_{0}^{*}, \xi_{1}^{*}, \cdots, \xi_{r}^{*}$. If, then, we specialize $0^{*}$ to the various affine rings $\boldsymbol{D}_{j}, \boldsymbol{D}_{j}=K\left[\xi_{0}^{*} / \xi_{j}^{*}, \xi_{1}^{*} / \xi_{j}^{*}, \cdots, \xi_{j-1}^{*} / \xi_{j}^{*}, \xi_{j+1}^{*} / \xi_{j}^{*}, \cdots, \xi_{n}^{*} / \xi_{j}^{*}\right]$, $j=0,1,2, \cdots, r$, it follows that in each the first $r$ coordinates are algebraically independent and the rest depend integrally on them. It is well known that if $\mathfrak{B}$ is a prime divisor of $\Sigma$ such that $\mathfrak{B}(\mathfrak{B})$ contains two of the rings $\boldsymbol{D}_{j}$, say $\mathfrak{o}_{\alpha}, \mathfrak{o}_{\beta} \subset \mathfrak{B}(\mathfrak{B})$, and if $\mathfrak{B}$ is of first kind with respect to $\mathfrak{o}_{\alpha}$, then $\mathfrak{B}$ is also of the first kind with respect to $\mathfrak{o}_{\beta}$. Consequently, one may speak of a prime divisor as being of first or second kind relative to a given projective model $V_{r}$

(2) Numbers in brackets refer to the bibliography at the end of this paper.

$\left({ }^{3}\right)$ By the center of $\mathfrak{P}$ in $\Im$ is meant the prime ideal in $\Im$ consisting of those elements $\eta$ such that $v(\mathfrak{B}, \eta)>0$. Throughout this paper we use the notation $v(\mathfrak{B}, x)$ to denote the value of $x$ in the valuation $\mathfrak{B}$. 
of $\Sigma$. Thus we may associate with a model $V_{r}$ of $\Sigma$ a set $\subseteq\left(V_{r}\right)$ of prime divisors, consisting of all those prime divisors which are of first kind with respect to $V_{r}$.

Using the elements of $\mathfrak{S}\left(V_{r}\right)$ as generators we form the abelian group $\mathfrak{B}\left(V_{r}\right)$ which consists of all finite, formal, power products $\mathfrak{P}_{1}^{\alpha_{1}} \mathfrak{B}_{2}^{\alpha_{2}} \cdots \mathfrak{P}_{k}^{\alpha_{k}}$ where $\mathfrak{B}_{i} \in \mathfrak{S}\left(V_{r}\right)$ and $\alpha_{i}$ is an integer, $i=1,2, \cdots, k$. The elements of (B) $\left(V_{r}\right)$ are called divisors. The divisor $\mathfrak{A}=\prod_{i=1}^{k} \mathfrak{P}_{i}^{\alpha_{i}}$ is said to be integral if $\alpha_{i}>0$, $i=1,2, \cdots, k$.

With every element $\eta$ in $\Sigma$ one can associate a uniquely determined divisor $\mathfrak{A}(\eta)$ in $\mathfrak{S}\left(V_{r}\right)$,

$$
\mathfrak{A}(\eta)=\prod \mathfrak{P}^{v(\eta)}, \quad v(\eta)=v(\mathfrak{B}, \eta),
$$

where the product is extended over all prime divisors of the set $\mathfrak{S}$. (When no ambiguity will result, we shall write $\eta$ instead of $\mathfrak{A}(\eta)$.) Those divisors which are associated in this way with elements $\eta$ in $\Sigma$ form a subgroup $\mathfrak{S}$ of $\mathfrak{S}\left(V_{r}\right)$. Two divisors $\mathfrak{A}$ and $\mathfrak{B}$ are said to be equivalent if $\mathfrak{A} \cdot \mathfrak{B}^{-1} \in \mathfrak{S}$.

With every prime divisor $\mathfrak{B}$ in $\mathfrak{S}$ one can associate a unique, irreducible, $(r-1)$-dimensional subvariety, $V_{r-1}(\mathfrak{B})$, of $V_{r}$. With every integral divisor $\mathfrak{A}$ in $\mathfrak{S}\left(V_{r}\right)$, say $\mathfrak{A}=\prod \mathfrak{P}_{i}^{\alpha_{i}}$, we associate the effective subvariety

$$
V(\mathfrak{U})=A=\alpha_{1} V\left(\mathfrak{B}_{1}\right)+\alpha_{2} V\left(\mathfrak{P}_{2}\right)+\cdots+\alpha_{k} V\left(\mathfrak{B}_{k}\right) .
$$

The complete system determined by $\mathfrak{A}$ (or $A$ ) is, then, the set of all effective subvarieties $V\left(\mathfrak{A}^{\prime}\right)$ which are associated with integral divisors $\mathfrak{X}^{\prime}$ which are equivalent to $\mathfrak{A}$.

In what is to follow, we shall need the following lemma, proved in [4].

Lемма 1.1 The algebraically independent quantities $\xi_{1}, \xi_{2}, \cdots, \xi_{r}$ $\left(\xi_{i}=\xi_{i}^{*} / \xi_{0}^{*}, i=1,2, \cdots, r\right)$ have divisor representations of the form $\xi_{i}=\mathfrak{A}_{i} / \mathfrak{A}_{0}$, $i=1,2, \cdots, r$, where $\mathfrak{A}_{i}$ and $\mathfrak{A}_{0}$ are integral divisors and no prime divisor divides both $\mathfrak{A}_{i}$ and $\mathfrak{A}_{0}$.

The following lemma is also needed.

LemMa 1.2. If $\mathfrak{P}$ is a prime factor of $\mathfrak{A}_{0}$, say $\mathfrak{A}_{0}=\mathfrak{B}^{\beta} \mathfrak{A}_{0}^{\prime}$ where $\mathfrak{A}_{0}^{\prime}$ is prime to $\mathfrak{B}$, and if $f\left(\xi_{1}, \xi_{2}, \cdots, \xi_{r}\right)$ is any polynomial in $\xi_{1}, \xi_{2}, \cdots, \xi_{r}$ of degree $h$, then $v(\mathfrak{B}, f)=-h \beta$. (The notation is that of the previous lemma.)

Proof. Assume first of all that $f=\phi_{h}$, a homogeneous polynomial in $\xi_{1}, \xi_{2}, \cdots, \xi_{r}$ of degree $h$, so that $\phi_{h} / \xi_{1}^{h}=\phi_{h}\left(1, \xi_{2} / \xi_{1}, \cdots, \xi_{r} / \xi_{1}\right)$. This element is in ${D_{1}}_{1}$, and since $\mathfrak{P}$ is of first kind, and at finite distance with respect to $D_{1}$, the elements $\xi_{2} / \xi_{1}, \xi_{3} / \xi_{1}, \cdots, \xi_{r} / \xi_{1}$ must be algebraically independent modulo $\mathfrak{p}_{1}$, the center of $\mathfrak{P}$ in $\mathfrak{0}_{1}$. Hence $v\left(\mathfrak{P}, \phi_{h} / \xi_{1}^{h}\right)=0$ and $v\left(\mathfrak{P}, \phi_{h}\right)=h v\left(\mathfrak{P}, \xi_{1}\right)$ $=-h \beta$. 
If $f$ is an arbitrary polynomial then the lemma follows for $f$ also since $v(\mathfrak{B}, a+b)=\min (v(\mathfrak{B}, a), v(\mathfrak{B}, b))$ if $v(\mathfrak{B}, a) \neq v(\mathfrak{B}, b)$.

It is not difficult to see that if $\omega$ is any integral function of $\xi_{1}, \xi_{2}, \cdots, \xi_{r}$ then $\omega$ admits a divisor representation of the form

$$
\omega=\mathfrak{B} / \mathfrak{A}_{0}^{k}
$$

where $\mathfrak{B}$ is an integral divisor and $k$ is an integer. In fact, if $\omega$ is an integral function of $\xi_{1}, \xi_{2}, \cdots, \xi_{r}$, then $\omega$ has non-negative value at any prime divisor at which the values of $\xi_{1}, \xi_{2}, \cdots, \xi_{r}$ are non-negative. Conversely, any element $\omega$ in $\Sigma$ which admits a representation of the form $\omega=\mathfrak{B} / \mathfrak{A}_{0}^{k}$ is an integral function of $\xi_{1}, \xi_{2}, \cdots, \xi_{r}$.

2. Exponents of integral functions. We now show that the DedekindWeber method of assigning exponents to integral functions can be carried over to the case of functions of several variables almost verbatim. The real significance of these exponents is intimately connected with the notion of arithmetically normal varieties, and we shall treat the subject from this point of view.

Let $\xi_{0}^{*}, \xi_{1}^{*}, \cdots, \xi_{n}^{*}$ be the homogeneous coordinates of the general point of a variety $V_{r}$ in the projective space $P_{n}$. It is assumed that $V_{r}$ is arithmetically normal so that $\mathrm{D}^{*}=K\left[\xi_{0}^{*}, \xi_{1}^{*}, \cdots, \xi_{n}^{*}\right]$ is integrally closed in its quotient field $\Sigma^{*}$. As in the preceding section we assume that $\xi_{0}^{*}, \xi_{1}^{*}, \cdots, \xi_{r}^{*}$ are algebraically independent and that $0^{*}$ depends integrally on these $r+1$ elements. We again consider the rings $\mathrm{D}_{0}, \mathrm{D}_{1}, \cdots, \mathrm{D}_{r}, \mathrm{D}_{i}=K\left[\xi_{0}^{*} / \xi_{i}^{*}, \cdots, \xi_{i-1}^{*} / \xi_{i}^{*}\right.$, $\left.\xi_{i+1}^{*} / \xi_{i}^{*}, \cdots, \xi_{n}^{*} / \xi_{i}^{*}\right]$. If $\lambda$ is any element in $\mathfrak{D}_{0}$, then $\lambda$ is a polynomial $f\left(\xi_{1}, \xi_{2}, \cdots, \xi_{n}\right)\left(\xi_{i}=\xi_{i}^{*} / \xi_{0}^{*}\right)$ of degree $h$, say. Consequently, $\xi_{0}^{* h} \cdot \lambda=\lambda^{*}$ is an element of $0^{*}$ which is homogeneous of degree $h$. It is pointed out in [9] that any element $\lambda^{*}$ in $0^{*}$ which is homogeneous of degree $h$ satisfies an equation of the form

$$
\lambda^{* \nu}+a_{1}\left(\xi_{0}^{*}, \cdots, \xi_{r}^{*}\right) \lambda^{*-1}+\cdots+a_{\nu}\left(\xi_{0}^{*}, \cdots, \xi_{r}^{*}\right)=0
$$

where $a_{i}$ is a form in $\xi_{0}^{*}, \xi_{1}^{*}, \cdots, \xi_{r}^{*}$ of degree $h \cdot i, i=1,2, \cdots, \nu$ $\left(\nu=\left[\Sigma^{*}: K\left(\xi_{0}^{*}, \xi_{1}^{*}, \cdots, \xi_{r}^{*}\right)\right]\right)$. If we divide equation $(2.1)$ by $\xi_{i}^{* h \nu}$, $i=0,1, \cdots, r$, we find

$$
\left(\lambda^{*} / \xi_{i}^{* h}\right)^{\nu}+\left(a_{1} / \xi_{i}^{* h}\right)\left(\lambda^{*} / \xi_{i}^{* h}\right)^{\nu-1}+\cdots+a_{\nu} / \xi_{i}^{* h \nu}=0 .
$$

In other words $\lambda^{*} / \xi_{i}{ }^{* h}\left(=\lambda / \xi_{i}^{h}\right)$ is integrally dependent upon $\boldsymbol{D}_{i}$ and hence is in $\boldsymbol{D}_{i}$. We thus see that given any element $\lambda$ in $D_{0}$ there exist integers $h$ such that $\lambda / \xi_{i}^{h} \subset_{0_{i}}, i=1,2, \cdots, r$. Clearly, if this is true for one integer $h$ then it is true for any integer $k$ greater than $h$. If for some $i$, the integer $h$ is such that $\lambda / \xi_{i}^{h} \subset \mathrm{o}_{i}$, then it is also true that $\lambda / \xi_{j}^{h} \subset_{0_{j}}$, for any $j=1,2, \ldots, r_{r}$. In fact, since $\lambda$ is in $D_{0}$, we have 


$$
\lambda=\mathfrak{B} / \mathfrak{A}_{0}^{k}
$$

where $\mathfrak{B}$ is integral, and

$$
\lambda / \xi_{i}^{h}=\left(\mathfrak{B} / \mathfrak{A}_{0}^{k}\right) \cdot\left(\mathfrak{A}_{0}^{h} / \mathfrak{A}_{i}^{h}\right)=\mathfrak{B} / \mathfrak{A}_{0}^{k-h} \cdot \mathfrak{A}_{i}^{h} .
$$

If $k$ is the smallest integer such that (2.2) holds (that is, if $\mathfrak{B}$ is not a multiple of $\mathfrak{\Re}_{0}$ ), then the fact that $\lambda / \xi_{i}^{h} \subset \mathfrak{o}_{i}$ implies that $h \geqq k$, for $\lambda / \xi_{i}^{h} \subset \mathfrak{D}_{i}$, implies that $\lambda / \xi_{i}^{h}$ may be written in the form

$$
\lambda / \xi_{i}^{h}=\mathfrak{M} / \mathfrak{A}_{i}^{s}
$$

where $\mathfrak{M}$ is integral. Equations (2.3) and (2.4) then imply that $\mathfrak{A}_{0}^{k-1} \cdot \mathfrak{P}_{i}^{h} \cdot \mathfrak{M}$ $=\mathfrak{B} \mathfrak{H}_{i}^{s}$ and, since $\mathfrak{A}_{i}$ and $\mathfrak{A}_{0}$ are relatively prime, $k \leqq h$ for $\mathfrak{B}$ is not divisible by $\mathfrak{A}_{0}$. Since we can represent $\lambda$ in the form $\lambda=\mathfrak{B}^{\prime} / \mathfrak{A}_{0}^{h}$ it follows that $\lambda / \xi_{j}^{h}=\mathfrak{B}^{\prime} / \mathfrak{R}_{j}^{h}$, and hence that $\lambda / \xi_{j}^{h} \subset \mathfrak{o}_{j}$.

We therefore define the exponent of the function $\lambda$ (notation: $\exp \lambda$ ) to be the smallest integer $h$ such that $\lambda / \xi_{i}^{h} \subset_{0_{i}}$. The exponent depends only upon $\lambda$ and not upon the particular $\xi_{i}$ which we take to define it. In the case of one independent quantity, $\xi_{1}$, this definition is precisely that of Dedekind-Weber.

Several obvious, but important, properties of exponents suggest themselves immediately. We list them below.

(a) $\exp \lambda=h$ if and only if $\lambda=\mathfrak{B} / \mathfrak{A}_{0}^{h}$, where $\mathfrak{B}$ is an integral divisor not di. visible by $\mathfrak{A}_{0}$.

In fact, if $\lambda=\mathfrak{B} / \mathfrak{P}_{0}^{h}$ then clearly $\exp \lambda \leqq h$, but if $\exp \lambda=h^{\prime}<h$, then $\lambda=\mathfrak{B}^{\prime} / \mathfrak{A}_{0}^{h^{\prime}}$ as has been shown. This implies that $\mathfrak{B}$ is divisible by $\mathfrak{A}_{0}$, which is not the case.

(b) As a corollary to (a) we have $\exp \lambda=0$ if and only if $\lambda \in K$.

(c) If $\eta$ and $\zeta$ are two elements of $\mathrm{D}_{0}$, then $\exp (\eta+\zeta) \leqq \max (\exp \eta, \exp \zeta)$, and if $\exp \eta>\exp \zeta$ then $\exp (\eta+\zeta)=\exp \eta$.

If $\exp \eta=h$ and $\exp \zeta=s$, and if $h \geqq s$ then $\eta / \xi_{1}^{h}$ and $\zeta / \xi_{1}^{h}$ are in $0_{1}$. Hence $\exp (\eta+\zeta) \leqq h$. If $h>s$, then $(\eta+\zeta) / \xi_{1}^{h-1}$ is not in $0_{1}$ for if it were then we would have $\eta / \xi_{1}^{h-1}=(\eta+\zeta) / \xi^{h-1}-\zeta / \xi_{1}^{h-1}$ in $D_{1}$, which is not the case.

(d) If $\lambda \in \mathbb{0}_{0}$ and $\exp \lambda=h$, then $\lambda$ is a polynomial in $\xi_{1}, \xi_{2}, \cdots, \xi_{n}$ of degree less than or equal to $h$.

This statement holds in view of the fact that $V$ is a normal model of $\Sigma$ and hence the system of hyperplane sections on $F$ and all of its multiples are complete [4].

(e) If $\lambda=f\left(\xi_{1}, \xi_{2}, \cdots, \xi_{n}\right)$ is a polynomial of degree $h$ then $\exp \lambda \leqq h$.

(f) If $\eta$ and $\zeta$ are in $\mathrm{D}_{0}$, then $\exp (\eta \cdot \zeta) \leqq \exp \eta+\exp \zeta$. If $\eta$ is a polynomial in the independent elements $\xi_{1}, \xi_{2}, \cdots, \xi_{r}$ only, then $\exp (\eta \cdot \zeta)=\exp \eta+\exp \zeta_{\text {. }}$

The first statement follows directly from (a); the second fellows from (a) in view of Lemma (1.2).

(g) If $\mathfrak{A}_{0}$ is a prime divisor $\mathfrak{P}$ then the equality sign always holds in (f). Moreover, $\exp \lambda=-v(\mathfrak{B}, \lambda)$. 
(h) If $\lambda^{*}=\phi\left(\xi_{0}^{*}, \xi_{1}^{*}, \cdots, \xi_{n}^{*}\right)$ is an element of $0^{*}$ of degree of homogeneity $h$ then $\exp \lambda\left(\lambda=\phi / \xi_{0}^{* h}\right)$ is less than $h$ if and only if $\phi$ is divisible by $\xi_{0}^{*}$ in $0^{*}$.

Assertion (h) follows almost immediately from (d) and (e). If $\exp \lambda=k<h$ then, by (d), $\lambda$ is a polynomial of degree less than or equal to $k$ in $\xi_{1}, \xi_{2}, \cdots, \xi_{n}$, $\lambda=f\left(\xi_{1}, \xi_{2}, \cdots, \xi_{n}\right)$, say. Consequently $\psi=\xi_{0}^{* k} f$ is an element of $0^{*}$, and $\phi=\xi_{0}^{* h-k} \psi$. On the other hand, if $\phi=\xi_{0}^{* \sigma} \phi^{\prime}, \sigma>0, \phi^{\prime}$ in $0^{*}$, then $\lambda=\phi / \xi_{0}^{* h}$ $=\phi^{\prime} / \xi_{0}{ }^{* h-\sigma}=g\left(\xi_{1}, \xi_{2}, \cdots, \xi_{h}\right)$ where $g$ is a polynomial of degree $h-\sigma$. By (e) this implies $\exp \lambda \leqq h-\sigma$.

THEOREM 2.1. If $\mathrm{o}^{*}$ has an independent modular base over $K\left[\xi_{0}^{*}, \xi_{1}^{*}, \cdots, \xi_{r}^{*}\right]$ consisting of $\nu$ homogeneous elements $\lambda_{1}{ }^{*}, \lambda_{2}{ }^{*}, \cdots, \lambda_{\nu}{ }^{*}$ where $\nu=\left[\Sigma: K\left(\xi_{1}, \cdots, \xi_{r}\right)\right]$ then $\mathrm{D}_{0}$ (and of course also $\mathrm{o}_{j}, j=0,1, \cdots, r$ ) possesses a $D W$-base over $K\left[\xi_{1}, \xi_{2}, \cdots, \xi_{r}\right]$ and conversely.

Proof. Assume that the elements $\lambda_{1}{ }^{*}, \lambda_{2}^{*}, \cdots, \lambda_{\nu}{ }^{*}$ are arranged in the order of increasing degree, so that if $\lambda_{i}{ }^{*}$ is homogeneous of degree $r_{i}$ we have $r_{1} \leqq r_{2} \leqq \cdots \leqq r_{v}$. If $\omega^{*}$ is any homogeneous element of $0^{*}$ of degree $h$, we have

$$
\omega^{*}=P_{1}^{*} \lambda_{1}^{*}+P_{2}^{*} \lambda_{2}^{*}+\cdots+P_{\nu}^{*} \lambda_{\nu}^{*}, P_{j}^{*} \in K\left[\xi_{0}^{*}, \xi_{1}^{*}, \cdots, \xi_{r}^{*}\right] .
$$

If $P_{i}^{*}=\sum P_{i j}^{*}$, where $P_{i j}^{*}$ is homogeneous of degree $j$, it follows immediately by an application of the automorphism $\tau: \xi_{0}^{*} \rightarrow t \xi_{0}^{*}$ of $\Sigma^{*}$ over $\Sigma$ $\left(\Sigma^{*}=K\left(\xi_{0}^{*}, \xi_{1}^{*}, \cdots, \xi_{n}^{*}\right)\right.$ that $P_{i j}^{*} \equiv 0$ if $j \neq h-r_{i}$, and that $P_{i}^{*}=P_{i h-r_{i}}^{*}$. In other words, in the representation (2.5) of the homogeneous element $\omega^{*}$, the coefficient $P_{i}^{*}$ of $\lambda_{i}^{*}$ is homogeneous of degree $h-r_{i}$.

It follows from the above remarks that none of the elements $\lambda_{i}^{*}$ is divisible by $\xi_{0}^{*}$ in $0^{*}$. In fact, suppose $\lambda_{i}^{*}=\xi_{0}^{*} \omega^{*}$. Since $\omega^{*}$ is of degree $r_{i}-1$ (we assume for the moment that $i>1$ ) we have $\omega^{*}=P_{1}^{*} \lambda_{1}^{*}+\cdots+P_{i-1}^{*} \lambda_{i-1}^{*}$, and hence $\lambda_{i}^{*}=Q_{1}^{*} \lambda_{1}^{*}+\cdots+Q_{i-1}^{*} \lambda_{i-1}^{*}$, where $Q_{j}^{*}=\xi_{0}^{*} P_{j}^{*}$. This is in contradiction with the independence of the elements $\lambda_{i}^{*}$. In particular, $\lambda_{1}^{*}$ must be a constant, which we may take to be 1 .

If we let $\lambda_{i}=\lambda_{i}^{*} / \xi_{0}^{* r_{i}}$, it follows that $\lambda_{i}$ is an element of $D_{0}$ of exponent $r_{i}$. If $\omega$ is an element of $\boldsymbol{D}_{0}$ of exponent $h$, then $\xi_{0}{ }^{* h} \omega$ is homogeneous of degree $h$, and is in $\left.0^{*}{ }^{4}\right)$. Hence we have $\xi_{0}{ }^{* h} \omega=P_{1}^{*} \lambda_{1}{ }^{*}+P_{2}{ }^{*} \lambda_{2}^{*}+\cdots+P_{\nu}^{*} \lambda_{\nu}{ }^{*}$ where $P_{i}^{*}$ is homogeneous of degree $h-r_{i}$. Consequently, we have $\omega=\sum P_{i} \lambda_{i}$, where $P_{i}=P_{i}^{*} / \xi_{0}^{* h-r_{i}}$. This implies of course that $\exp P_{i}\left(=\right.$ degree of $P_{i}$ ) is less than or equal to $h-r_{i}$.

Since this argument could be carried out with any of the rings $\boldsymbol{D}_{j}$ as well as with $D_{0}$, it follows that the elements $\lambda_{1}, \lambda_{2}, \cdots, \lambda_{\nu}$ have the properties of a DW-base. (See introduction.)

Conversely, if $\lambda_{1}, \lambda_{2}, \cdots, \lambda_{\nu}$ form a DW-base in $\boldsymbol{o}_{0}$ relative to the ring

(4) This assertion follows by property (d) above. In fact, since exp $\omega=h, \omega$ is a polynomial of degree $h$ in $\xi_{1}, \xi_{2}, \cdots, \xi_{n}$ and hence $\xi_{0}{ }^{* h} \omega$ is a form of degree $h$ in $\xi_{0}^{*}, \xi_{1}^{*}, \cdots, \xi_{n}^{*}$. 
$K\left[\xi_{1}, \xi_{2}, \cdots, \xi_{r}\right]$ and if $\exp \lambda_{i}=r_{i}$ it follows immediately that the elements $\lambda_{i}^{*}=\xi_{0}^{* r} \lambda_{i}$ form a modular base for $0^{*}$ over $K\left[\xi_{0}^{*}, \xi_{1}^{*}, \cdots, \xi_{r}^{*}\right]$, q.e.d.

3. Differential divisors and the geometric genus. We shall henceforth assume that we are dealing with a field $\Sigma$ of degree of transcendency two over the coefficient field $K$. We use the same notations as before except that we put $r=2$. We select the quantities $\xi_{0}^{*}, \xi_{1}^{*}, \xi_{2}^{*}$ in such a way that in addition to our previous requirements we also have $\left[\Sigma: K\left(\xi_{1}, \xi_{2}\right)\right]=\nu$, the order of our model $F$. (We put $\xi_{i}=\xi_{i}^{*} / \xi_{0}^{*}, i=1,2, \cdots, n$.)

Let $\Delta$ be any subfield of $\Sigma$ such that $\Sigma$ is algebraic over $\Delta$. If $\mathfrak{B}$ is any prime divisor of $\Sigma$ with valuation ring $\mathfrak{B}$, then $\mathfrak{B}_{\Delta}=\mathfrak{B} \cap \Delta$ is a valuation ring in $\Delta$ which defines a prime divisor $\mathfrak{B}_{\Delta}$ in $\Delta$. The divisor $\mathfrak{P}_{\Delta}$ is the valuation which $\mathfrak{P}$ induces in $\Delta$. If $\mathfrak{p}_{\Delta}$ is the ideal of non-units in $\mathfrak{B}_{\Delta}$, and $\mathfrak{p}$ is the ideal of non-units in $\mathfrak{B}$, then the extended ideal $\mathfrak{p}_{\Delta} \cdot \mathfrak{B}$ is a power of $\mathfrak{p}$, say $\mathfrak{p}_{\Delta} \cdot \mathfrak{B}=\mathfrak{p}^{e}, e \geqq 1$. The divisor $\mathfrak{P}$ is said to be ramified with respect to the field $\Delta$ if $e>1$, unramified if $e=1$. The integer $e-1$ is called the ramification degree of $\mathfrak{P}$ relative to $\Delta$.

We restrict ourselves to the case in which $\Delta=K\left(\xi_{1}, \xi_{2}\right)$. We consider the ideal $Z_{\left(\xi_{1}, \xi_{2}\right)}=$ g.c.d. $(\partial f / \partial z)_{z=\omega}[9]$, where $\omega$ is an arbitrary element of $\boldsymbol{o}_{0}$ and $f\left(z ; \xi_{1}, \xi_{2}\right)=N(z-\omega)$. If $\mathfrak{p}_{1}, \mathfrak{p}_{2}, \cdots, \mathfrak{p}_{s}$ are the one-dimensional prime ideals in $\boldsymbol{D}_{0}$ which occur in the decomposition of $Z_{\left(\xi_{1}, \xi_{2}\right)}$ then we have

$$
Z_{\left(\xi_{1}, \xi_{2}\right)} \approx \mathfrak{p}_{1}^{\alpha_{1}} \cdot \mathfrak{p}_{2}^{\alpha_{2}} \cdots \mathfrak{p}_{s}^{\alpha_{s}}
$$

where the symbol $\approx$ denotes quasi-equality in the sense of van der Waerden. It is well known that if $\mathfrak{P}$ is ramified with respect to $\Delta$, and if the $v$-ring $\mathfrak{B}$ of $\mathfrak{B}$ contains $\mathfrak{D}_{0}$, then $\mathfrak{p}$, the center of $\mathfrak{P}$ in $\mathfrak{D}_{0}$, is one of the primes $\mathfrak{p}_{1}, \mathfrak{p}_{2}, \cdots, \mathfrak{p}_{s}$ and conversely. Moreover, the ramification degree of $\mathfrak{B}$ relative to $\Delta$ is precisely the exponent $\alpha$ assigned to its center in the decomposition (3.1). We conclude therefore that there are only a finite number of prime divisors in the set $\subseteq(F)$ which are ramified over the field $\Delta\left(=K\left(\xi_{1}, \xi_{2}\right)\right)$.

The assertions of the preceding paragraph are all consequences of the following lemma.

LEMMA 3.1. If $\mathfrak{B}$ is any prime divisor of the field $\Sigma$ (at finite distance relative to the ring $\left.\mathrm{D}_{0}\right)$ of ramification degree $e-1$ relative to $K\left(\xi_{1}, \xi_{2}\right)$, then there is a primitive element $\theta$ in $\mathrm{D}_{0}$ such that $f^{\prime}(\theta) \equiv 0\left(\mathfrak{p}^{(e-1)}\right), f^{\prime}(\theta) \not \equiv\left(\mathfrak{p}^{(e)}\right)$, where $\mathfrak{p}$ is the center of $\mathfrak{B}$ in $\mathrm{D}_{0}$, and $f^{\prime}(\theta)=(\partial f / \partial z)_{z=\theta}$. Moreover, it may be assumed that $\theta$ has the form $\theta=c_{0}+c_{1} \xi_{1}+c_{2} \xi_{2}+\cdots+c_{n} \xi_{n}, c_{i} \in K$.

Proof. The proof is similar to the considerations set forth in [10, paragraphs 11,12 , and 13]. We assume that $\xi_{1}$ is transcendental mod $\mathfrak{P}$ so that the residue field of $\mathfrak{P}$ is algebraic over the field $K\left(\xi_{1}\right)$. We let $L$ denote the least normal (Galoisian) extension of $K\left(\xi_{1}\right)$ which contains the residue field of $\mathfrak{B}$. Together with $\mathfrak{D}_{0}$ we consider the rings $\mathfrak{0}_{0}^{\prime}=K\left(\xi_{1}\right)\left[\xi_{2}, \xi_{3}, \cdots, \xi_{n}\right]$ and $\mathfrak{D}_{0}=L\left[\xi_{2}, \xi_{3}, \cdots, \xi_{n}\right]$. In view of the fact that $\xi_{1}$ is transcendental mod $\mathfrak{p}$ 
there is one and only one prime ideal $\mathfrak{p}^{\prime}$ in $\mathfrak{D}_{0}^{\prime}$ such that $\mathfrak{p}^{\prime} \cap \mathfrak{o}_{0}=\mathfrak{p}$. Moreover, if $\Im^{\prime}$ is the quotient ring of $0_{0}^{\prime}$ at $\mathfrak{p}^{\prime}$ and $\Im$ that of ${D_{0}}_{0}$ at $\mathfrak{p}$ then clearly $\Im=\Im^{\prime}$ and consequently the ramification degree of $\mathfrak{B}$ as a "point" of the one-dimensional variety corresponding to $D_{0}^{\prime}$ is the same as the ramification degree of $\mathfrak{B}$ as a "curve" of the variety belonging to $\mathfrak{D}_{0}$. Now if $\mathfrak{a}_{1}, \mathfrak{a}_{2}, \cdots, \mathfrak{a}_{h}$ are the prime ideals in $\mathfrak{O}_{0}$ which lie over $\mathfrak{p}^{\prime}$, that is, $\mathfrak{a}_{i} \cap \mathfrak{o}_{0}^{\prime}=\mathfrak{p}^{\prime}$, then $\mathfrak{D}_{0} \mathfrak{p}^{\prime}$ $=\left[\mathfrak{a}_{1}, \mathfrak{a}_{2}, \cdots, \mathfrak{a}_{h}\right]$. Moreover, it follows immediately from the first part of the proof of Theorem 7 in [10] that if $m$ is any non-negative integer then $\mathfrak{a}_{i}^{m} \cap \mathrm{D}_{0}^{\prime}=\mathfrak{p}^{\prime m}(5)$.

Consider the field $L\left(\xi_{2}\right) \supset K\left(\xi_{1}, \xi_{2}\right)$, and let $P$ be the divisor induced by $\mathfrak{B}$ in $K\left(\xi_{1}, \xi_{2}\right)$. The divisor $\mathfrak{A}_{i}$ in $L \cdot \Sigma=\Lambda$ defined by $\mathfrak{a}_{i}$ in $\mathfrak{D}_{0}$ induces a divisor $A_{i}$ in $L\left(\xi_{2}\right)$ which lies over $P$ in $K\left(\xi_{1}, \xi_{2}\right)$. Let $p, a_{i}$ be the centers of $P$ and $A_{i}$ in their respective $v$-rings, and let $A_{i}$ be the center of $\mathfrak{A}_{i}$ in its $v$-ring. If $\Re_{i}$ is the $v$-ring of $\mathfrak{A}_{i}$ then we assert that $a_{i} \cdot \Re_{i}=\mathcal{A}_{i}^{e}$ where $e-1$ is the ramification degree of $\mathfrak{P}$ relative to $K\left(\xi_{1}, \xi_{2}\right)$. In other words, the ramification degree of $\mathfrak{A}_{i}$ relative to $L\left(\xi_{2}\right)$ is the same as that of $\mathfrak{B}$ relative to $K\left(\xi_{1}, \xi_{2}\right)$. To see this, let $R$ be the $v$-ring of $P$ in $K\left(\xi_{1}, \xi_{2}\right)$ and let $S_{i}$ be that of $A_{i}$ in $L\left(\xi_{2}\right)$. Now $a_{i}=p S_{i}$ and hence $a_{i} \cdot \Re_{i}=p \cdot \Re_{i}$. On the other hand, $\Re_{i}=\Im^{\prime} \cdot \Re_{i}$ and $p \cdot \Re_{i}=\left(p \cdot \Im^{\prime}\right) \cdot \Re_{i}$ $=A^{\prime} \bullet \cdot \Re_{i}$ where $A^{\prime}$ denotes the center of $\mathfrak{B}$ in $\mathfrak{Y}^{\prime}$. Since $\mathcal{A} \cdot \mathcal{A}^{\prime} \cdot \Re_{i}=A_{i}$ we have $A^{\prime} e \cdot \Re_{i}=A_{i}^{e}$. This implies the assertion.

The lemma under consideration is well known in the case of functions of one variable. In fact, if $\theta=c_{1} \xi_{1}+c_{2} \xi_{2}+\cdots+c_{n} \xi_{n}, c_{i} \in L$, is a primitive element of $\Lambda$ over $L\left(\xi_{2}\right)$ then for non-special "constants" $c_{i}$, we will have $f^{\prime}(\theta) \equiv 0\left(\mathfrak{a}_{i}^{e-1}\right), f^{\prime}(\theta) \not \equiv 0\left(\mathfrak{a}_{\mathfrak{i}}^{e}\right)$. Since the values of $c_{i}$ that must be avoided are those satisfying certain algebraic relations, we can, in particular, find such quantities in $K$. For such a choice of the $c$ 's, $\theta$ will be an element of $\boldsymbol{D}_{0}$ and so also will $f^{\prime}(\theta)$. This proves the lemma in view of the fact that $\mathfrak{a}_{\mathfrak{i}}^{m} \cap \mathrm{D}_{0}=\mathfrak{p}^{(m)}$, which holds, in particular, for $m=e-1, e$.

The ramification divisor $B_{\Delta}$ of $\Sigma$ relative to $\Delta$ is defined by the relation

$$
\mathfrak{3}_{\Delta}=\prod \mathfrak{P}^{e-1}, \quad \mathfrak{B} \in \mathfrak{S},
$$

where $e-1$ is the ramification degree of $\mathfrak{B}$ and the product is extended over all prime divisors of the set $\mathfrak{S}(F)$. $\mathfrak{Z}_{\Delta}$ may of course contain prime factors which are also factors of $\mathfrak{A}_{0}$. ( $\mathfrak{A}_{0}$ is the common denominator of $\xi_{1}$ and $\xi_{2}$ mentioned in Lemma 1.1. It is given by the curve $\xi_{0}^{*}=0$ on $F$.)

We let $\xi_{0}$ denote the complementary module of $\mathfrak{D}_{0}$ relative to $K\left(\xi_{1}, \xi_{2}\right)$. $\xi_{0}$ consists of those elements $\epsilon$ in $\Sigma$ such that $S(\epsilon \omega)$ is integral for every $\omega$ in $\mathfrak{D}_{0} . S(\epsilon \omega)$ denotes the trace of $\epsilon \omega$ relative to $K\left(\xi_{1}, \xi_{2}\right)$. The module $\xi_{0}$ is of

(5) The methods of $[10$, Theorem 7] are applicable here in view of the fact that as a minimal prime ideal of the integrally closed ring $D_{0}, \mathfrak{p}$ defines a simple subvariety of the model $V_{2}$ associated with $\mathfrak{D}_{0}$ and hence $\mathfrak{p}^{\prime}$ defines a simple subvariety (point) of the model $V_{1}^{\prime}$ associated with $\mathfrak{D}_{0}^{\prime}$. 
paramount importance in the theory of ramified divisors. We prove that $\xi_{0}$ is the inverse system of the ideal $Z_{\left(\xi_{1}, \xi_{2}\right)}$. To this end two lemmas are needed, the first of which will be used later in another connection.

LEMMA 3.2. If $\Delta=K\left(\xi_{1}, \xi_{2}\right)$ and $\mathfrak{B}$ is any prime divisor of the set $\mathfrak{S}(F)$, and if $\mathfrak{B}_{1}(=\mathfrak{B}), \mathfrak{B}_{2}, \cdots, \mathfrak{B}_{k}$ are the complete set of prime divisors in $\Sigma$ which induce the same valuation in $\Delta$ as does $\mathfrak{B}$, then for any function $\eta$ such that $v\left(\mathfrak{B}_{i}, \eta\right)>0, i=1,2, \cdots, k$, it is true that $v(\mathfrak{B}, S(\eta))>0$, where the trace is taken relative to $\Delta$.

Proof. Let $P$ be the common contraction of $\mathfrak{P}_{1}, \mathfrak{P}_{2}, \cdots, \mathfrak{P}_{k}$ with $\Delta$ and let $\Omega$ be the least normal extension of $\Delta$ which contains $\Sigma$. If $P_{1}, P_{2}, \cdots, P_{s}$ denote the various valuations of $\Omega$ which induce the valuation $P$ on $\Delta$, then each of these induces one of the valuations $\mathfrak{P}_{i}$ on $\Sigma, i=1,2, \cdots, k$. The intersection $B_{1} \cap B_{2} \cap \cdots \cap B_{s}$ where $B_{i}$ is the $v$-ring of $P_{i}$ is the integral closure (in $\Omega$ ) of the $v$-ring $\mathscr{B}$ of $P$ in $\Delta[3]$. We denote this intersection by $D$. The ring $D$ contains $s$ distinct maximal prime ideals $\mathfrak{a}_{1}, \mathfrak{a}_{2}, \cdots, \mathfrak{a}_{8}$ where $\mathfrak{a}_{i}$ consists of those elements $\delta$ in $D$ with the property $v\left(\mathscr{P}_{i}, \delta\right)>0$. By hypothesis, $\eta$ is contained in the intersection $a=\left[\mathfrak{a}_{1}, \mathfrak{a}_{2}, \ldots, \mathfrak{a}_{8}\right]$. Since the ideals $\mathfrak{a}_{1}, \mathfrak{a}_{2}, \cdots, \mathfrak{a}_{8}$ are conjugate over $\Delta[3]$, the intersection $\mathfrak{a}$ is an invariant ideal, and hence not only $\eta$ but also all of its conjugates are in a. Hence their sum $S(\eta)$ is in a. Since $\mathfrak{a} \cap \mathfrak{B}$ is the ideal of non-units in $\mathfrak{B}$, we conclude that $v(P, S(\eta))>0$, q.e.d.

LEMмA 3.3. An element $\epsilon$ of $\Sigma$ is an element of the module $\xi_{0}$ if and only if $v(\mathfrak{B}, \epsilon) \geqq-e+1$ for every prime divisor of the set $\mathfrak{S}(F)$ whose v-ring contains $\mathrm{D}_{0}$ $(e-1$ denotes the ramification degree of $\mathfrak{P})$.

Proof. Let $\epsilon$ be an element of $\Sigma$ with this property. If $\epsilon$ is not an element of $\mathcal{E}_{0}$ then there is an element $\omega$ in $D_{0}$ such that $S(\epsilon \omega)$ is not integral. If we restrict our attention to the group $\mathrm{S}_{\Delta}$ of divisors in $\Delta$ generated by the prime divisors in $\Delta$ of first kind with respect to the rings $K\left[\xi_{1}, \xi_{2}\right], K\left[1 / \xi_{1}, \xi_{2} / \xi_{1}\right]$, and $K\left[\xi_{1} / \xi_{2}, 1 / \xi_{2}\right]$, then $S(\epsilon \omega)$ will have a decomposition of the form

$$
S(\epsilon \omega)=\left(\left(\prod_{i=1}^{h} P_{i}^{\alpha_{i}}\right) /\left(\prod_{j=1}^{k} Q_{j}^{\beta_{j}}\right)\right) A_{0}^{\sigma}
$$

where $\alpha_{i}, \beta_{j}>0, \sigma$ is an integer, $P_{i}$ and $Q_{j}$ are distinct prime divisors in $\Delta$, and $A_{0}$ is that divisor in $\Delta$ which corresponds to $\mathfrak{A}_{0}$ in $\Sigma$, that is, $\xi_{i}=A_{i} / A_{0}$, $i=1,2$.

We fix our attention on one of the divisors $Q_{i}$, say $Q_{1}$. If $q_{1}$ is the center of $Q_{1}$ in $R\left(=K\left[\xi_{1}, \xi_{2}\right]\right)$, then $q_{1}$ is a principal ideal, say $q_{1}=R \cdot \phi$, where $\phi$ is a polynomial in $\xi_{1}, \xi_{2}$. We consider the extended ideal $0_{0} q_{1}$ which has a decomposition of the form $\mathfrak{D}_{0} q_{1}=\left[\mathfrak{p}_{1}^{\left(f_{1}\right)}, \mathfrak{p}_{2}^{\left(f_{2}\right)}, \cdots, \mathfrak{p}_{s}^{\left(f_{s}\right)}\right]$. By definition, it follows that $f_{i}-1$ is the ramification degree of the prime divisor of $\Sigma$ determined by $\mathfrak{p}_{i}$. 
Now by our hypothesis we have $v\left(\mathfrak{p}_{i}, \epsilon \omega \phi\right) \geqq-f_{i}+1+f_{i}$ (since $v\left(\mathfrak{p}_{i}, \omega\right) \geqq 0$ ), and hence $v\left(\mathfrak{p}_{i}, \epsilon \omega \phi\right)>0, i=1,2, \cdots, s$. By Lemma 3.2 it follows that $v\left(Q_{1}, S(\epsilon \omega \phi)\right)>0$, or $v\left(Q_{1}, S(\epsilon \omega)\right)+1>0$, and hence we have $v\left(Q_{1}, S(\epsilon \omega)\right) \geqq 0$, which is a contradiction. We therefore conclude that $\epsilon$ is an element of $\mathfrak{F}_{0}$.

Conversely, it is well known (see for example [5]) that for any primitive element $\theta$ in $\mathfrak{D}_{0}$ the relation $f^{\prime}(\theta) \cdot \bigotimes_{0} \subset \mathfrak{o}_{0}$, where $f(z)=N(z-\theta)$, holds. Moreover, by Lemma 3.1 it follows that for any prime divisor $\mathfrak{B}$ with center $\mathfrak{p}$ in $D_{0}$ and ramification degree $e-1$ there exists a primitive element $\theta$ in $D_{0}$ such that $f^{\prime}(\theta) \equiv 0\left(p^{(e-1)}\right), f^{\prime}(\theta) \neq \equiv 0\left(p^{(e)}\right)$. From this it follows that for any $\epsilon$ in $\mathfrak{F}_{0}$ the inequality $v(\mathfrak{B}, \epsilon) \geqq-e+1$ holds. This proves the lemma.

This characterization of $\mathbb{F}_{0}$ in terms of the ramified divisors at finite distance relative to $\mathfrak{D}_{0}$ (that is, having $v$-rings which contain $\mathfrak{D}_{0}$ ) is obviously equivalent to the statement that $\xi_{0}$ is the inverse system of $Z_{\left(\xi_{1}, \xi_{2}\right)}$ in $\Sigma$, in view of the relation (3.1). Making use of this fact, it is not difficult to see that every element $\epsilon$ of $\mathbb{E}_{0}$ admits a divisor representation of the form

$$
\epsilon=\left(\mathfrak{D} / \mathfrak{Z}_{\Delta}\right) \mathfrak{A}_{0}^{r}
$$

where $\mathfrak{D}$ is an integral divisor and $r$ is an integer which may be positive, negative, or zero $\left.{ }^{6}\right)$. Conversely, any element of $\Sigma$ which admits a representation such as (3.3) is in $\xi_{0}$.

An integral divisor $\mathfrak{D}$ equivalent to the divisor $3_{\Delta} \mathfrak{A}_{0}^{-3}$ (in symbols $\mathfrak{D} \sim \mathfrak{Z}_{\Delta} \mathfrak{A}_{0}^{-3}$ ) is called a differential divisor of first kind. To every such divisor $\mathfrak{D}$ there corresponds an element $\delta$, necessarily in $\mathbb{E}_{0}$, which expresses the equivalence, namely,

$$
\delta=\mathfrak{D} \mathfrak{A}_{0}^{3} / \mathfrak{B}_{\Delta} .
$$

The geometric genus $p_{o}$ of the surface $F$ (a normal nonsingular model) is defined as the number of linearly independent differential divisors of first kind, that is the number of linearly independent elements $\delta$ in $\xi_{0}$ of the form (3.4). A curve $K$ on $F$ determined by a differential divisor of first kind is called a canonical curve, and the complete system $|K|$ is called the pure canonical system on $F$. The effective dimension of $|K|$ is $p_{0}-1$.

(6) Equation (3.3) follows immediately if none of the prime factors of $\mathfrak{A}_{0}$ is ramified. Otherwise, the proof is as follows. If $\mathfrak{B}$ is any prime factor of $\mathfrak{A}_{0}$, say $\mathfrak{A}_{0}=\mathfrak{P}^{e} \cdot \mathfrak{A}_{0}^{\prime}$, where $\mathfrak{A}_{0}^{\prime}$ is prime to $\mathfrak{B}$, then $e-1$ is the ramification degree of $\mathfrak{P}$. Since $\mathfrak{P}$ is at finite distance relative to the ring $D_{1}\left(=K\left[1 / \xi_{1}, \xi_{2} / \xi_{1}, \cdots, \xi_{n} / \xi_{1}\right]\right)$ it follows that there exists an element $\theta_{1}$ in $D_{1}$ of exponent one, such that $f^{\prime}\left(\theta_{1}\right) \equiv 0\left(\mathfrak{p}^{(e-1)}\right), f^{\prime}\left(\theta_{1}\right) \not \equiv\left(\mathfrak{p}^{(e)}\right)$ where $\mathfrak{p}$ is the center of $\mathfrak{B}$ in $\mathbb{D}_{1}$. Since $\theta_{1}$ is of exponent one it follows that $\theta=\xi_{1} \theta_{1}$ is in $D_{0}$. A simple computation leads to the conclusion that $v\left(\mathfrak{B}, f^{\prime}(\theta)\right)=-e(\nu-1)+e-1$, so that in the representation $f^{\prime}(\theta)=\mathfrak{R} \mathbb{B}_{\Delta} / \mathscr{A}_{0}{ }^{\nu-1}$ the divisor $\mathfrak{R}$ does not have $\mathfrak{P}$ as a factor. Since $\epsilon \cdot f^{\prime}(\theta)=\mathfrak{M} / \mathfrak{R}_{0}^{k}$, where $\mathfrak{M}$ is integral, we have $\epsilon=\left(\mathfrak{M} / \mathbb{R} \mathfrak{Z}_{\Delta}\right) \mathfrak{A}_{0}{ }^{\nu-k-1}$. Applying this process to each of the factors of $\mathfrak{A}_{0}$ in turn, we find that $\epsilon=\left(\mathfrak{N} / \mathfrak{U} \mathfrak{Z}_{\Delta}\right) \mathfrak{R}_{0}^{r}$ where $\mathfrak{U}$ has no factors in common with $\mathfrak{A}_{0}$. It then follows by Lemma 3.3 that $\mathfrak{N}$ contains $\mathfrak{U}$ as a factor. 
As it stands, our definition of differential divisors of first kind and of the geometric genus $p_{0}$ is but a formal generalization of similar definitions given by Dedekind and Weber in [2]. However, it is not difficult to see that if one considers a generic projection of our normal nonsingular model $F$ into a surface in $S_{3}$ given by $f\left(\xi_{1}, \xi_{2}, \theta\right)=0$ where $\theta=c_{0}+c_{1} \xi_{1}+\cdots+c_{n} \xi_{n}$ then the elements $\delta f^{\prime}(\theta)$ will be adjoint polynomials, $\phi_{\nu-4}$ of degree $\nu-4$, to the surface $f\left(\xi_{1}, \xi_{2}, \theta\right)=0$. Hence since $\delta=\phi_{\nu-4} / f^{\prime}(\theta)$ the $p_{o}$ independent elements $\delta$ determine the $p_{o}$ independent double integrals of first kind attached to $F$.

4. The arithmetic genus. Let $x_{0}, x_{1}, \cdots, x_{n}$ be indeterminates and let $P$ denote the prime $H$-ideal in $K\left[x_{0}, x_{1}, \cdots, x_{n}\right]$ which determines a normal, nonsingular model $F$ of $\Sigma$. In other words, $P$ is a prime ideal in $K\left[x_{0}, x_{1}, \cdots, x_{n}\right]$ such that $K\left[x_{0}, x_{1}, \cdots, x_{n}\right] / P \cong_{0^{*}}$ and $x_{i} \rightarrow \xi_{i}{ }^{*}$ under the isomorphism. Van der Waerden has shown [7] that if $\chi(P, h)$ denotes the number of homogeneous elements of degree $h$ in $K\left[x_{0}, x_{1}, \cdots, x_{n}\right]$ which are linearly independent (over $K$ ) $\bmod P$, then for large values of $h$ (that is, for $h>h_{0}$, where $h_{0}$ is a fixed integer depending on $\left.P\right) \chi(P, h)$ is given by

$$
\chi(P, h)=a_{0} C_{h, 2}+a_{1} C_{h, 1}+a_{2}
$$

where $a_{0}, a_{1}$, and $a_{2}$ are integers independent of $h$. Van der Waerden showed further that $a_{0}=\nu$, the order of the surface $F$.

Formula (4.1) can also be derived by means of the Riemann-Roch theorem for surfaces, with the help of Noether's formulae for the genus and order of a composite curve [8]. The theorem of Riemann-Roch states that if $|D|$ is a complete linear system on a surface $F$ then its dimension $r$ is given by the formula $r=n-\pi+p_{a}+1-i+s$ where $n, \pi$, and $i$ are respectively the degree, genus, and index of speciality of $|D|$, and $p_{a}$ is the arithmetic genus of $F$. The non-negative integer $s$ is called the superabundance of the system, and the difference $s-i$ is called the irregularity of $|D|$. The system $|D|$ is said to be regular if $s=i=0$. It has been shown by Severi, that if $|C|$ is a nonsingular system of dimension $r \geqq 3$, then for large values of $h$ the system $\left|C_{h}\right|=|h C|$ is a regular system. Applying the Riemann-Roch theorem to $\left|C_{h}\right|$, and denoting its dimension, degree and genus by $\rho_{h}, \nu_{h}$ and $\pi_{h}$ respectively, we have

$$
\rho_{h}=\nu_{h}-\pi_{h}+p_{a}+1 .
$$

If $F$ is a normal, nonsingular model of the field $\Sigma$, and $|C|$ is its system of hyperplane sections, then $|C|$ is nonsingular, and the complete system $|h C|$ is cut out on $F$ by the hypersurfaces of order $h$ in its ambient space [4]. Hence in this case, $\chi(P, h)=\rho_{h}+1$. A straightforward application of Noether's formulae to formula (4.2) yields

$$
\chi(P, h)=(h(h-1) / 2) \nu+(\nu-\pi+1) h+p_{a}+1,
$$


where $\nu$ is the order of $F$ and $\pi$ is the genus of $|C|\left(^{7}\right)$.

As yet, no purely arithmetic proof of formula (4.2) is available. For the purposes of this paper we shall regard formula (4.3) as the definition of $\left.p_{a}{ }^{8}\right)$.

5. The existence of a DW-base implies $p_{g}=p_{a}$. Let $0^{*}=K\left[\xi_{0}^{*}, \xi_{1}^{*}, \cdots, \xi_{n}^{*}\right]$ where $\xi_{0}{ }^{*}, \xi_{1}^{*}, \cdots, \xi_{n}^{*}$ are the homogeneous coordinates alcng a normal nonsingular model $F$ of the field $\Sigma$. We make the following assumptions:

(a) $\xi_{0}^{*}, \xi_{1}^{*}, \xi_{2}^{*}$ are algebraically independent and every element of $0^{*}$ depends integrally on them.

(b) There exist $\nu$ ( $=$ order of $F$ ) homogeneous elements $\lambda_{1}^{*}, \lambda_{2}{ }^{*}, \cdots, \lambda_{\nu}{ }^{*}$ in $0^{*}$ linearly independent over $K\left[\xi_{0}^{*}, \xi_{1}^{*}, \xi_{2}^{*}\right]$ with the property that if $\omega^{*}$ is any element in $0^{*}$ then $\omega^{*}=P_{1}^{*} \lambda_{1}^{*}+P_{2}^{*} \lambda_{2}^{*}+\cdots+P_{\nu}^{*} \lambda_{\nu}^{*}$, where $P_{i}^{*}$ is a polynomial in $\xi_{0}^{*}, \xi_{1}^{*}, \xi_{2}^{*}$. We let $r_{i}$ be the degree of $\lambda_{i}^{*}$, and we assume as we did in Theorem 2.1 that $r_{1}=0,1 \leqq r_{2} \leqq r_{3} \leqq \cdots \leqq r_{v}$.

In the course of the proof of Theorem 2.1 we saw that under the above assumptions we could assert in addition that if $\omega^{*}$ is a homogeneous element of $0^{*}$ of degree $h$, and if, say, $r_{s}$ is the last integer in the sequence $r_{1}, r_{2}, \cdots, r_{\nu}$ such that $h \geqq r_{s}$ (that is, $h<r_{s+1}$ if $\lambda_{s+1}^{*}$ is defined), then $\omega^{*}=P_{1}^{*} \lambda_{1}^{*}+P_{2}^{*} \lambda_{2}^{*}+\cdots$ $+P_{s}^{*} \lambda_{s}^{*}$ where $P_{i}^{*}$ is a homogeneous form in $\xi_{0}^{*}, \xi_{1}^{*}, \xi_{2}^{*}$ of degree $h-r_{i}$.

The function $\chi(P, h)$ mentioned in the preceding section gives the number of homogeneous elements in $0^{*}$ of degree $h$ which are linearly independent over $K$. If in particular $h>r$, we know that any homogeneous element of degree $h$ can be written in the form $P_{1}^{*} \lambda_{1}^{*}+P_{2}^{*} \lambda_{2}^{*}+\cdots+P_{\nu}^{*} \lambda_{\nu}^{*}$ where $P_{i}^{*}$ is homogeneous of degree $h-r_{i}$. Since $\lambda_{1}^{*}, \lambda_{2}{ }^{*}, \cdots, \lambda_{v}^{*}$ are linearly independent over $K\left[\xi_{0}^{*}, \xi_{1}^{*}, \xi_{2}^{*}\right]$ it follows that a linearly independent $K$-base for the homogeneous elements of degree $h$ in $0^{*}$ can be obtained by taking together all of the elements of the $\nu$ sets of elements of the form $a_{i}^{(j)} \lambda_{i}{ }^{*}$ where $a_{i}^{(j)}$ runs over a linearly independent base for the homogeneous polynomials in $\xi_{0}^{*}, \xi_{1}^{*}, \xi_{2}{ }^{*}$ of degree $h-r_{i}$. There are, then, $(1 / 2)\left(h-r_{i}+1\right)\left(h-r_{i}+2\right)$ such elements in the $i$ th set $\left\{a_{i}^{(j)} \lambda_{i}^{*}\right\}$ and hence we have

$$
\chi(P, h)=\sum_{i=1}^{\nu} \frac{\left(h-r_{i}+1\right)\left(h-r_{i}+2\right)}{2} .
$$

A simple computation (taking into account that $r_{1}=0$ ) yields

(7) For a complete discussion of these questions see [8, chapter 4].

(8) It seems to us that from the arithmetic point of view, formula (4.3) is a very natural way in which to introduce the arithmetic genus. The question might be raised as to the necessity of requiring $F$ to be nonsingular as well as normal in order that formula (4.3) hold. The cone projecting an elliptic cubic from a point not in the plane of the cubic furnishes an example of a surface in $S_{3}$ which is arithmetically normal but on which the complete system $\left|C_{h}\right|$ cut out by the surfaces of order $h$ in $S_{3}$ has a superabundance $s=+1$ for every $h$. 


$$
\begin{aligned}
\chi(P, h)=\frac{h(h-1)}{2} \nu & +\left(\nu-\sum_{i=2}^{\nu}\left(r_{i}-1\right)+1\right) h \\
& +\sum_{i=2}^{\nu} \frac{\left(r_{i}-1\right)\left(r_{i}-2\right)}{2}+1
\end{aligned}
$$

Combining (5.1) and (4.3) we conclude that

$$
p_{a}=\sum_{i=2}^{\nu} \frac{\left(r_{i}-1\right)\left(r_{i}-2\right)}{2} .
$$

The proof that $p_{g}=p_{a}$ is completed by showing that $p_{g}$ can also be expressed in terms of the integers $r_{i}$, and that the expression one thereby obtains is $p_{0}=(1 / 2) \sum_{i=2}^{v}\left(r_{i}-1\right)\left(r_{i}-2\right)$. This is somewhat more difficult.

As was pointed out in Theorem 2.1, if $\lambda_{i}=\lambda_{i}^{*} / \xi_{0}^{*^{*} i}$ then $\lambda_{1}, \lambda_{2}, \cdots, \lambda_{\nu}$ is a DW-base in $\boldsymbol{D}_{0}$ relative to the ring $K\left[\xi_{1}, \xi_{2}\right]$. It is well known that there exist $\nu$ elements $\epsilon_{1}, \epsilon_{2}, \cdots, \epsilon_{\nu}$ in $\Sigma$ satisfying the equations $S\left(\epsilon_{i} \lambda_{j}\right)=\delta_{i j}$ $\left(\delta_{i i}=1, \delta_{i j}=0, i \neq j\right)$. These elements $\epsilon$ are all in $\xi_{0}$ (the complementary modulo of $D_{0}$ ) and, in fact, form a modular base for $\xi_{0}$ over $K\left[\xi_{1}, \xi_{2}\right]$, called the complementary base to $\lambda_{1}, \lambda_{2}, \cdots, \lambda_{\nu}$. The elements $\epsilon_{1}, \epsilon_{2}, \cdots, \epsilon_{\nu}$ are of course linearly independent over $K\left(\xi_{1}, \xi_{2}\right)$. If $\epsilon$ is any element of $\xi_{0}$, then we have $\epsilon=a_{1} \epsilon_{1}+a_{2} \epsilon_{2}+\cdots+a_{\nu} \epsilon_{\nu}, a_{i} \in K\left[\xi_{1}, \xi_{2}\right]$, and it follows from the equaticn $S\left(\epsilon_{i} \lambda_{j}\right)=\delta_{i j}$ that the coefficient $a_{i}$ is equal to $S\left(\epsilon \lambda_{i}\right)$.

We assume, in particular, that $\epsilon$ is of the form $\mathfrak{D} \mathfrak{A}_{0}^{3} / \mathfrak{Z}_{\Delta}$ and we assert that if $\mathfrak{B}$ is any factor of $\mathfrak{A}_{0}$, then $v\left(\mathfrak{B}, \xi_{1}^{2} \epsilon\right)>0$. This is obvious if $\mathfrak{P}$ is not a factor of $3_{\Delta}$. If, on the other hand, $Z_{\Delta}=\mathfrak{B}^{\beta} \mathfrak{B}^{\prime}\left(\mathfrak{Z}^{\prime}\right.$ prime to $\left.\mathfrak{B}\right), \beta>0$, then since $\mathfrak{B}$ induces the divisor $P$ in $K\left(\xi_{1}, \xi_{2}\right)$ at which $\left(1 / \xi_{1}\right)$ has positive value, it follows from the definitions of $\mathfrak{Z}_{\Delta}$ and $\mathfrak{A}_{0}$ that $v\left(\mathfrak{B}, 1 / \xi_{1}\right)=\beta+1$, and that $\mathfrak{A}_{0}=\mathfrak{P}^{\beta+1} \mathfrak{A}_{0}^{\prime}$. We conclude that $v(\mathfrak{B}, \epsilon) \geqq 3(\beta+1)-\beta=2 \beta+3$ and hence that $v\left(\mathfrak{P}, \xi_{1}^{2} \epsilon\right) \geqq 2 \beta+3-2(\beta+1)=1$.

Writing $\epsilon$ in the form $\epsilon=\sum a_{i} \epsilon_{i}$ we have

$$
a_{i}=S\left(\epsilon \lambda_{i}\right), \quad a_{i} / \xi_{1}^{r_{i}-2}=S\left(\xi_{1}^{2} \epsilon \cdot \lambda_{i} / \xi_{1}^{r_{i}}\right) .
$$

Since $\lambda_{i} / \xi_{1}^{r_{i}}$ is an element of $\mathrm{D}_{1}\left(\mathrm{o}_{1}=K\left[\xi_{0}^{*} / \xi_{1}^{*}, \xi_{2}^{*} / \xi_{1}^{*}, \cdots, \xi_{n}^{*} / \xi_{1}^{*}\right]\right)$ its value at any of the prime factors of $\mathfrak{A}_{0}$ is non-negative, and hence the value of $\xi_{1}^{2} \epsilon \lambda_{i} / \xi_{1}^{r_{i}}$ is positive at any such factor. It follows by Lemma 3.2 that $v\left(\mathfrak{B}, S\left(\xi_{1}^{2} \epsilon \lambda_{i} / \xi_{1}^{\tau_{i}}\right)\right)>0$, and hence by (5.4), $v\left(\mathfrak{B}, a_{i} / \xi_{1}^{r_{i}-2}\right)>0$ for any such prime factor $\mathfrak{B}$. By Lemma 1.2, the inequality $v\left(\mathfrak{B}, a_{i} / \xi_{1}^{r_{i}-2}\right)>0$ implies that if $r_{i} \leqq 2$ then $a_{i}$ is identically zero, and if $r_{i}>2$ the degree of $a_{i}$ is less than $r_{i}-2$.

We prove the converse of this result. That is, if $\epsilon=\sum a_{i} \epsilon_{i}$ is an element of $\mathfrak{G}_{0}$ such that $a_{i} \equiv 0$ if $r_{i} \leqq 2$, and for those values of $i$ for which $r_{i}>2$ the degree 
of $a_{i}$ is less than $r_{i}-2$ then the element $\epsilon$ admits a divisor decomposition of the form $\epsilon=\mathfrak{D} \mathfrak{A}_{0}^{3} / \mathfrak{Z}_{\Delta}$. We consider the set of elements $\lambda_{1}^{\prime}, \lambda_{2}^{\prime}, \cdots, \lambda_{\nu}^{\prime}$ where $\lambda_{i}^{\prime}=\lambda_{i} / \xi_{1}^{\gamma_{i}}$. As we have seen, these elements form a DW-base for the ring $D_{1}$. It is well known that the base $\epsilon_{1}^{\prime}, \epsilon_{2}^{\prime}, \cdots, \epsilon_{\nu}^{\prime}$ complementary to $\lambda_{1}^{\prime}, \lambda_{2}^{\prime}, \cdots, \lambda_{\nu}^{\prime}$ is related to the base $\epsilon_{1}, \epsilon_{2}, \cdots, \epsilon_{\nu}$ by the equations $\epsilon_{i}^{\prime}=\xi_{1}^{r_{i}} \epsilon_{i}$. These elements form an independent modular base over $K\left[1 / \xi_{1}, \xi_{2} / \xi_{1}\right]$ for the complementary module $\xi_{1}$ of $D_{1}$ relative to the ring $K\left[1 / \xi_{1}, \xi_{2} / \xi_{1}\right]$.

Let $\mathfrak{P}$ be any factor of $\mathfrak{A}_{0}$, say $\mathfrak{A}_{0}=\mathfrak{B}^{e} \mathfrak{A}_{0}^{\prime}$ where $\mathfrak{A}_{0}^{\prime}$ is prime to $\mathfrak{B}$. If $e>1$, we have $\mathfrak{B}^{e-1}$ as a factor of $\mathfrak{Z}_{\Delta}$. Since $\mathfrak{B}$ is a divisor at finite distance in the coordinate system which gives rise to $D_{1}$, and since $\epsilon_{i}^{\prime}$ is in $\xi_{1}$ it follows that $v\left(\mathfrak{B}, \epsilon_{i}^{\prime}\right) \geqq-e+1$. Hence we see that $v\left(\mathfrak{B},\left(1 / \xi_{1}\right) \epsilon_{i}^{\prime}\right) \geqq e+(-e+1)=1$. We thus have $v\left(\mathfrak{B}, \xi_{1}^{r_{i}-1} \epsilon_{i}\right)>0$ and $v\left(\mathfrak{B}, \epsilon_{i}\right)>e\left(r_{i}-1\right)$. By our assumptions concerning the coefficients $a_{i}$ we have $v\left(\mathfrak{B}, a_{i}\right) \geqq-e\left(r_{i}-3\right)$, and hence we conclude that $v\left(\mathfrak{B}, a_{i} \epsilon_{i}\right)>2 e$.

The element $a_{i} \epsilon_{i}$ is in $\xi_{0}$, and if $a_{i}$ is not identically zero we can write

$$
a_{i} \epsilon_{i}=\left(\mathfrak{M} / \mathfrak{B}_{\Delta}\right) \mathfrak{B}_{1}^{s_{1}} \cdot \mathfrak{B}_{2}^{s_{2}} \cdots \mathfrak{P}_{k}^{s_{k}}
$$

where $\mathfrak{M}$ is an integral divisor and $\mathfrak{P}_{1}, \mathfrak{P}_{2}, \cdots, \mathfrak{B}_{k}$ are the factors of $\mathfrak{A}_{0}$. If $\left(e_{j}-1\right)$ is the ramification degree of $\mathfrak{B}_{j}$ then by our above results we have $s_{1}-\left(e_{j}-1\right)>2 e_{j}$, and hence $s_{1} \geqq 3 e_{j}$. Hence, since $\mathfrak{A}_{0}=\prod_{j} \mathfrak{B}_{j}^{e_{j}}$, we conclude that the element $a_{i} \epsilon_{i}$ is of the form

$$
a_{i} \epsilon_{i}=\mathfrak{R}_{0}^{3} \mathfrak{N} / \Re_{\Delta} .
$$

The integral divisor $\mathfrak{N}$ is therefore a differential divisor of first kind. The number of independent elements of the form $a_{i} \epsilon_{i}$ in $\mathcal{E}_{0}$ which give rise to differential divisors of first kind is therefore zero if $r_{i} \leqq 2$ and $\left(r_{i}-1\right)\left(r_{i}-2\right) / 2$ (=number of independent $a_{i}$ of degree less than $r_{i}-2$ ) if $r_{i}>2$. Since the $\epsilon_{i}$ form a base for $\mathfrak{G}_{0}$, we conclude that

$$
p_{o}=\sum_{r_{i} \geqq 3} \frac{\left(r_{i}-1\right)\left(r_{i}-2\right)}{2}=\sum_{i=2}^{\nu} \frac{\left(r_{i}-1\right)\left(r_{i}-2\right)}{2} .
$$

This completes the proof that the existence of a base implies $p_{g}=p_{a}$.

6. A lemma of Castelnuovo. This section is devoted to establishing an important lemma due originally to Castelnuovo [1]. We consider a normal, nonsingular model $F$ of $\Sigma$, and we use the same notations as before. In particular, we consider the various complete systems $\left|C_{h}\right|=|h C|$ cut out on $F$ by the hypersurfaces of order $h$ of its ambient space $P_{n}$.

The system $\left|C_{h}\right|$ cuts out a linear series $g_{\nu h}$ on a generic curve $C$ of the system of hyperplane sections $|C|$. (In what sense $C$ is generic will be specified in a moment.) The series $g_{\nu h}$ has a certain index of speciality $i_{h}$ and a certain deficiency $\delta_{h}$. Clearly, if $h$ is large we will have $i_{h}=0$. Moreover, since $C$ is 
generic in $|C|$ it is nonsingular and hence locally normal. This implies that $\delta_{h}=0$ for large values of $h$ [12]. The lemma of this section asserts the following equality $\left({ }^{9}\right)$

$$
p_{a}=\sum_{h=1}^{\infty}\left(i_{h}-\delta_{h}\right)
$$

Proof. We subject the homogeneous coordinates $\xi_{0}^{*}, \xi_{1}^{*}, \cdots, \xi_{n}^{*}$ to a projective transformation $\bar{\xi}_{i}=\sum a_{i j} \xi_{j}^{*}, a_{i j} \in K$. For non-special values of the $a_{i j}$ the property of integral dependence of $0^{*}$ upon the first three coordinates will not be lost. Moreover, since the system $|C|$ is obviously not composite with a pencil, it follows by the theorem of Bertini-Zariski [11] that for nonspecial values of the constants $a_{i j}$ the curve defined on $F$ by the equation $\bar{\xi}_{2}=0$ will be irreducible and nonsingular. In fact, it is easily seen from Zariski's consideration in [11] that for non-special constants $a_{i j}$ the ideal $0^{*} \bar{\xi}_{2}$ will be a prime $H$-ideal in $0^{*}$. (This of course involves the fact that $\mathrm{D}^{*}$ is integrally closed.) It is in this sense that we speak of the generic curve in $|C|$. We fix a set of constants $a_{i j}$ so that these requirements are satisfied. The characters $i_{h}$ and $\delta_{h}$ are understood to refer to the series $g_{\nu h}$ cut out on $\bar{\xi}_{2}=0$ by $\left|C_{h}\right|$.

As before we let $\chi(P, h)$ be the number of independent (over $K$ ) homogeneous elements of degree $h$ in $0^{*}$, and we let $\mu_{h}$ be the number of homogeneous elements of degree $h$ which are independent (over $K$ ) modulo $o^{*} \bar{\xi}_{2}$. It is not difficult to see that $\chi(P, h)=\mu_{h}+\mu_{h-1}+\cdots+\mu_{0}$. In fact, let $\theta_{1 i}, \theta_{2 i}, \cdots, \theta_{\mu_{i} i}$ be a set of $\mu_{i}$ homogeneous elements in $0^{*}$ of degree $i$ which are independent modulo $\mathrm{o}^{*} \bar{\xi}_{2}$. The $\sum_{i=0}^{h} \mu_{i}$ elements $\left\{\cdots, \bar{\xi}_{2}^{n-i} \theta_{1 i}, \bar{\xi}_{2}^{h-i} \theta_{2 i}\right.$, $\left.\cdots, \xi_{2}^{h-i} \theta_{\mu_{i} i}, \cdots\right\}$ are obviously independent, and every homogeneous element of degree $h$ in $0^{*}$ is a linear combination of these elements.

By the Riemann-Roch theorem (for curves), we have $\mu_{k}=k \nu-\pi+i_{k}$ $-\delta_{k}+1, k=1,2, \cdots, h$, where $\pi$ is the genus of $\bar{\xi}_{2}=0$, and since, clearly, $\mu_{0}=1$ we have

$$
\chi(P, h)=1+\sum_{k=1}^{h}\left\{k \nu-\pi+i_{k}-\delta_{k}+1\right\}
$$

or on rearranging (6.2) we have

$$
\chi(P, h)=\frac{h(h-1)}{2} \nu+(\nu-\pi+1) h+\sum_{k=1}^{h}\left(i_{k}-\delta_{k}\right)+1 .
$$

Since $\sum_{k=1}^{h}\left(i_{k}-\delta_{k}\right)$ remains constant for sufficiently large $h$ we see, if we compare (6.3) and (4.3), that $p_{a}=\sum_{k=1}^{\infty}\left(i_{k}-\delta_{k}\right)$, q.e.d.

(9) The original lemma of Castelnuovo [1] asserts that if one is given a system $|\Gamma|$, then for high values of $h$ the superabundance of $|h \Gamma|$ has a constant value $s$, and $s+p_{a}=\sum\left(i_{h}-\delta_{h}\right)$. 
7. On the indices $i_{h}$. We use the same notations and assumptions as in the preceding section. The index of speciality, $j_{h}$, of the system $\left|C_{h}\right|$ is defined as the dimension, increased by 1 , of the system $\left|K-C_{h}\right|$ where $|K|$ is the canonical system on $F$. If $\left|K-C_{h}\right|$ does not exist we put $j_{h}=0$. We observe first of all that $j_{h}=0$ if $h$ is sufficiently large.

The lemma of Severi [6] mentioned in the introduction is to the effect that if $h>\nu-4$ then the canonical system $|K|$, if it exists (that is, if $p_{o}>0$ ), cuts out a complete series on a generic curve $C_{h}$ of $\left|C_{h}\right|$. If $p_{o}=0$, the characteristic series $\left({ }^{10}\right)$ of $\left|C_{h}\right|$ is non-special. Since, in any case, the series cut out on $\left|C_{h}\right|$ by $|K|$ is totally contained in the series difference of the canonical series on $C_{h}$ and the characteristic series on $C_{h}$, it follows, by Severi's lemma, that for $h>\nu-4$ the complete series difference between the canonical and characteristic series on $C_{h}$ is cut out by the canonical system $|K|$. If in addition $h_{0}(>\nu-4)$ is taken large enough so that $j_{h_{0}}=0$, then for all $h \geqq h_{0}$ we have that the index of speciality of the characteristic series of $C_{h}$ is precisely $p_{0}$.

The formulas of Noether [8] yield the following two formulas immediately:

$$
\nu_{h}=h^{2} \nu_{1}, \quad \pi_{h}=h \pi_{1}+(h(h-1) / 2) \nu_{1}-(h-1)
$$

where $\nu_{h}$ and $\pi_{h}$ denote the degree and genus of $\left|C_{h}\right|$. From these one easily sees that if $h$ is sufficiently large, the inequality $\nu_{h}>\pi_{h}-1$ will hold.

Let $\rho$ be an integer greater than or equal to $h_{0}$ and in addition large enough so that for $h \geqq \rho, \nu_{h}>\pi_{h}-1$. We pass from $F$ to the derived normal surface $F_{\rho}$ of $F$ by referring the curves of $\left|C_{\rho}\right|$ to the sections of $F_{\rho}$ cut out by the hyperplanes of its ambient space $P_{N}$. Since $F$ is nonsingular, so is $F_{\rho}$ [9]. If $i_{k}$ is the index of speciality of the series cut out on a generic $C_{\dot{p}}$ by the system $\left|C_{k_{p}}\right|$, then

$$
i_{1}=p_{g}, i_{2}=i_{3}=\cdots=0 .
$$

(The condition $i_{2}=i_{3}=\cdots=0$ is implied by the fact that $k \nu_{\rho}>2 \pi_{\rho}-2$ for $k=2,3, \cdots$.) A model $F$ of $\Sigma$ will be called non-special if it is normal, nonsingular, and if the speciality indices on its generic hyperplane section satisfy equations (7.2).

We return to the notation of $\S 6$, but we now assume that the model $F$ considered there is non-special. Combining (6.1) and (7.2) we find

$$
\sum_{h=1}^{\infty} \delta_{h}=p_{o}-p_{a}
$$

It follows from (7.3) that if, in particular, $F$ is regular then $\delta_{h}=0$,

(10) By the characteristic series of $\left|C_{h}\right|$ on $C_{h}$ we mean, of course, the series cut out on the curve $C_{h}$ by the complete system $\left|C_{h}\right|$. 
$h=1,2, \cdots$. In other words, if $F$ is a non-special model of a regular field, then the systems $\left|C_{h}\right|$ cut out complete series on the general hyperplane section $C$ of $F$. This implies that the general hyperplane section of $F$ is arithmetically normal [4].

8. Construction of an independent base. Let $0^{*}=K\left[\xi_{0}^{*}, \xi_{1}^{*}, \cdots, \xi_{n}^{*}\right]$, where $\xi_{0}^{*}, \xi_{1}^{*}, \cdots, \xi_{n}^{*}$ are homogeneous coordinates along a non-special model $F$ of the field $\Sigma$. We assume that the quantities $\xi_{0}^{*}, \xi_{1}^{*}$ and $\xi_{2}^{*}$ are selected so that they are algebraically independent, and so that every element in $0^{*}$ depends integrally on them. The only other restriction which we place on the elements $\xi_{0}{ }^{*}, \xi_{1}{ }^{*}, \xi_{2}^{*}$ is that the net $N: c_{0} \xi_{0}{ }^{*}+c_{1} \xi_{1}{ }^{*}+c_{2} \xi_{2}{ }^{*}=0$ (the $c^{\prime}$ 's are parameters which vary over $K$ ) be sufficiently general in $|C|$ so that the general curve of $N$ will satisfy the condition of the preceding section, that is, we require that the general curve of $N$ be arithmetically normal. We let $R^{*}=K\left[\xi_{0}^{*}, \xi_{1}^{*}, \xi_{2}^{*}\right]$ and we shall prove that $\mathfrak{o}^{*}$ has an independent modular base over $R^{*}$.

We apply a nonsingular linear transformation $\eta_{i}=a_{i 0} \xi_{0}^{*}+a_{i 1} \xi_{1}^{*}+a_{i 2} \xi_{2}^{*}$, $a_{i j} \in K, i=0,1,2$, if necessary, in crder to insure that (a) the three ideals $0^{*} \eta_{0}, 0^{*} \eta_{1}, 0^{*} \eta_{2}$ will be prime in $0^{*}$, (b) that these curves will intersect pair by pair in $\nu$ distinct points of the surface $F$, each point of intersection being a simple intersection, (c) each of these curves will be arithmetically normal. Since $\xi_{0}^{*}, \xi_{1}^{*}, \xi_{2}^{*}$ are algebraically independent, it follows that the net $N$ is not composite with a pencil, and hence by [11] condition (a) is satisfied for a general choice of the constants $a_{i j} \in K$. It is easily seen that if the coefficients $a_{i j}$ are sufficiently general, conditions (b) and (c) will automatically be satisfied. We point out that since $\mathrm{o}^{*}$ depends integrally on $\eta_{0}^{*}, \eta_{1}{ }^{*}, \eta_{2}{ }^{*}$, the ideal $0^{*}\left(\eta_{0}^{*}, \eta_{1}^{*}, \eta_{2}^{*}\right)$ is projectively irrelevant, and hence none of the $\nu$ intersection points of $\eta_{1}=0$ and $\eta_{2}=0$ can be on the curve $\eta_{0}=0$.

It should be observed that the transformation from $\xi_{0}^{*}, \xi_{1}^{*}, \xi_{2}^{*}$ to $\eta_{0}, \eta_{1}, \eta_{2}$ does not change the ring $R^{*}$, and hence we may assume without loss of generality that the particular elements $\xi_{0}^{*}, \xi_{1}^{*}, \xi_{2}^{*}$ have all of the special properties which we have assigned to $\eta_{0}, \eta_{1}, \eta_{2}$.

We pass to the ring $D_{0}=K\left[\xi_{1}, \xi_{2}, \cdots, \xi_{n}\right]$ where $\xi_{i}=\xi_{i}^{*} / \xi_{0}^{*}$ and we observe that if $\mathfrak{A}=\mathfrak{D}_{0}\left(\xi_{1}, \xi_{2}\right)$ then the $K$-module $\mathfrak{D}_{0} / \mathfrak{A}$ is of rank $\nu$, the order of $F$. In fact if $\mathfrak{A}=\left[\mathfrak{q}_{1}, \mathfrak{q}_{2}, \cdots, \mathfrak{q}_{s}\right]$ is the primary decomposition of $\mathfrak{A}\left(\mathfrak{q}_{i}\right.$ is a zerodimensional ideal) then by assumption (b) we must have $s=\nu$ and $\mathfrak{q}_{i}=\mathfrak{p}_{i}$, $i=1,2, \cdots, \nu$, where $p_{i}$ is a prime zero-dimensional ideal. It follows immediately that $\mathfrak{A}^{*}=0^{*}\left(\xi_{1}^{*}, \xi_{2}^{*}\right)$ admits a primary decomposition of the form $\mathfrak{A}^{*}=\left[\mathfrak{p}_{1}^{*}, \mathfrak{p}_{2}^{*}, \cdots, \mathfrak{p}_{\nu}^{*}, \mathfrak{q}\right]$, where $\mathfrak{p}_{i}^{*}$ is the homogeneous prime in $\mathfrak{o}^{*}$ corresponding to the prime $\mathfrak{p}_{i}$ in $\mathfrak{p}_{0}$. As an ideal in $\mathfrak{D}^{*}, \mathfrak{p}_{i}^{*}$ is one-dimensional, but as a homogeneous ideal its dimension is counted as zero $\left.{ }^{11}\right)$. The ideal $\mathfrak{q}$ is a

(11) For a complete discussion of $H$-ideals and their related affine ideals, see the first two sections of van der Waerden [7]. 
possible embedded component of $\mathfrak{A}^{*}$ which is necessarily projectively irrelevant, that is, its associated prime in $0^{*}$ is the ideal $0^{*} \cdot\left(\xi_{0}^{*}, \xi_{1}^{*}, \cdots, \xi_{n}^{*}\right)$. The central point in preparing for the construction of a base is the following lemma.

Lemma 8.1. The fact that $\xi_{2}^{*}=0$ is an arithmetically normal curve on $F$ implies that $\mathfrak{H}^{*}$ has no irrelevant components.

Proof. If we let $\Im$ denote the ring $0^{*} / \xi_{2}^{*}$ then since $0^{*} \xi_{2}^{*}$ is prime, $\Im$ is an integral domain, and since $\xi_{2}^{*}=0$ is arithmetically normal, $\Im$ is integrally closed in its quotient field $\bar{\Sigma}$. This implies that if $\xi_{1}^{*} \rightarrow \bar{\xi}_{1} \bmod \xi_{2}^{*}$, then the principal ideal $\Im \bar{\xi}_{1}$ is an intersection of minimal ideals in $\Im$, and has no embedded ideals in $\Im$. Since $\mathfrak{A}^{*} / \xi_{2}^{*}=\Im \bar{\xi}_{1}$ it follows that $\mathfrak{A}^{*}$ can have no embedded ideals, q.e.d. We can thus write $\mathfrak{I}^{*}=\left[\mathfrak{p}_{1}^{*}, \mathfrak{p}_{2}^{*}, \cdots, \mathfrak{p}_{\nu}^{*}\right]$.

We now show that under the conditions we have specified above the construction of Dedekind-Weber can be generalized to the case now under consideration. Let $\omega_{1}, \omega_{2}, \cdots, \omega_{\nu}$ be $\nu$ elements of $D_{0}$ which are linearly independent modulo $\mathfrak{A}\left(=\mathbb{D}_{0}\left(\xi_{1}, \xi_{2}\right)\right)$. If $h_{i}$ is the smallest integer such that $\omega_{i}^{*}=\xi_{0}^{* h i} \omega_{i}$ is an element of $0^{*}$ then $\omega_{i}^{*}$ is homogeneous of degree $h_{i}$. Let us suppose that in $0^{*}$ a congruence of the form

$$
P_{1}\left(\xi_{0}^{*}\right) \omega_{1}^{*}+P_{2}\left(\xi_{0}^{*}\right) \omega_{2}^{*}+\cdots+P_{\nu}\left(\xi_{0}^{*}\right) \omega_{\nu}^{*} \equiv 0\left(\mathfrak{H}^{*}\right)
$$

is valid, where $P_{i}$ is a polynomial in $\xi_{0}^{*}$. Let $c_{1} \xi_{0}^{* k}$ be any term of $P_{1}$, so that $c_{1} \xi_{0}{ }^{* k} \omega_{1}^{*}$ is homogeneous of degree $s=k+h_{1}$. The homogeneous component of degree $s$ on the left-hand side of (8.1) will then be $c_{1} \xi_{0}^{* k} \omega_{1}^{*}$ $+c_{2} \xi_{0}{ }^{* s-h_{2}} \omega_{2}^{*}+\cdots+c_{\nu} \xi_{0}^{{ }^{*}-h_{\nu}} \omega_{\nu}^{*}$, where $c_{i} \xi_{0}^{* s-h_{i}}$ is a term of degree $s-h_{i}$ in $P_{i}$. Since $\mathfrak{I}^{*}$ is an $H$-ideal, we must have $\sum c_{i} \xi_{0}{ }^{* s-h}{ }^{*} \omega_{i}^{*}=A \xi_{1}^{*}+B \xi_{2}^{*}, A, B C_{0}{ }^{*}$. If $A=A_{m}+A_{m-1}+\cdots+A_{0}$ and $B=B_{m}+B_{m-1}+\cdots+B_{0}$, where $A_{i}$ and $B_{i}$ are forms of degree $i$ in $\xi_{0}^{*}, \xi_{1}^{*}, \cdots, \xi_{n}^{*}$ then under the automorphism $\tau: \xi_{0}^{*} \rightarrow t \xi_{0}^{*}, t \in K$ we obtain

$$
\begin{aligned}
t^{s}\left(A \xi_{1}^{*}+B \xi_{2}^{*}\right)= & t^{m+1}\left(A_{m} \xi_{1}^{*}+B_{m} \xi_{2}^{*}\right)+t^{m}\left(A_{m-1} \xi_{1}^{*}+B_{m-1} \xi_{2}^{*}\right) \\
& +\cdots+t\left(A_{0} \xi_{1}^{*}+B_{0} \xi_{2}^{*}\right) .
\end{aligned}
$$

Since this is an identity in $t, A \xi_{1}^{*}+B \xi_{2}{ }^{*}=A_{8-1} \xi_{1}^{*}+B_{s-1} \xi_{2}{ }^{*}$ and $\sum c_{i} \xi_{0}{ }^{* \sigma-h i}{ }^{k} \omega_{1}^{*}$ $=A_{s-1} \xi_{1}^{*}+B_{s-1} \xi_{2}^{*}$. If we divide this last equation by $\xi_{0}^{* s}$ we find $c_{1} \omega_{1}+c_{2} \omega_{2}$ $+\cdots+c_{\nu} \omega_{\nu} \equiv 0(\mathfrak{A})$. This implies $c_{1}=c_{2}=\cdots=c_{\nu}=0$. Repeating this process with the other homogeneous components on the left-hand side of (8.1) yields the conclusion that (8.1) implies $P_{1} \equiv P_{2} \equiv \cdots \equiv P_{\nu} \equiv 0$.

Conversely, if $\omega^{*}$ is an element of $0^{*}$ which is homogeneous of degree $s$, then $\omega^{*} / \xi_{0}^{* s}=\omega$ is in $D_{0}$ and $\omega-\left(c_{1} \omega_{1}+c_{2} \omega_{2}+\cdots+c_{\nu} \omega_{\nu}\right)=a \xi_{1}+b \xi_{2}, a$ and $b$ in $\mathfrak{D}_{0}$ and $c_{i} \in K, i=1,2, \cdots, \nu$. If $h$ is an integer so large that $\xi_{0}^{* h} \omega, \xi_{0}^{* h} \omega_{i}^{*}$, $\xi_{0}^{* h-1} a$ and $\xi_{0}^{* h-1} b$ are all in $0^{*}$ then we have 


$$
\xi_{0}^{* h-8} \omega^{*} \equiv \sum_{i=1}^{\nu} c_{i} \xi_{0}^{* h-h_{i}} \omega_{i}^{*}\left(\mathfrak{P}^{*}\right) .
$$

We select elements $\lambda_{1}{ }^{*}, \lambda_{2}{ }^{*}, \cdots, \lambda_{\nu}^{*}$ in $0^{*}$ as follows: take $\lambda_{1}{ }^{*}=1$, and let $\lambda_{2}{ }^{*}$ be an element in $\boldsymbol{D}^{*}$ of lowest degree of homogeneity, $r_{2}$, such that $\lambda_{2}{ }^{*}$ does not satisfy a congruence of the form $\xi_{0}^{* k-r_{2}} \lambda_{2}{ }^{*} \equiv c_{1} \xi_{0}^{* k-r_{1}} \lambda_{1}{ }^{*}\left(\mathfrak{U}^{*}\right)$, where $r_{1}=0$ is the degree of $\lambda_{1}^{*}$. We let $\lambda_{3}{ }^{*}$ be a homogeneous element of lowest degree in $0^{*}$, say $r_{3}$, such that $\lambda_{3}^{*}$ does not satisfy a congruence of the form $\xi_{0}^{* k-r_{3}} \lambda_{3}{ }^{*} \equiv c_{1} \xi_{0}^{* k-r_{1}} \lambda_{1}^{*}+c_{2} \xi_{0}^{* k-r_{2}} \lambda_{2}^{*}\left(\mathfrak{A}^{*}\right), c_{i} \in K$, for any integer $k$. We must have $r_{2} \leqq r_{3}$, for any element $\omega^{*}$ in $0^{*}$ of degree $h<r_{2}$ satisfies a congruence of the form $\xi_{0}{ }^{* k-h} \omega^{*} \equiv c_{1} \xi_{0}^{* k-r_{1}} \lambda_{1}^{*}\left(\mathfrak{A}^{*}\right)$. In general, if $\lambda_{1}^{*}, \lambda_{2}^{*}, \cdots, \lambda_{i}^{*}$ have been selected, and they are of degrees $r_{1}(=0), 1 \leqq r_{2} \leqq r_{3} \leqq \cdots \leqq r_{i}$ respectively, then we let $\lambda_{i+1}^{*}$ be an element of smallest degree of homogeneity, $r_{i+1}$, which does not satisfy a congruence of the form

$$
\xi_{0}^{* k-r_{i+1}} \lambda_{i+1}^{*} \equiv c_{1} \xi_{0}^{* k-r_{1}} \lambda_{1}^{*}+\cdots+c_{i} \xi_{0}^{* k-r_{i}} \lambda_{i}^{*}\left(\mathfrak{A}^{*}\right), \quad c_{i} \in K,
$$

for any integer $k$. We of course have $r_{i+1} \geqq r_{i}$.

This process yields exactly $\nu$ functions $\lambda_{1}^{*}, \lambda_{2}^{*}, \cdots, \lambda_{\nu}^{*}$ in $0^{*}$. This statement follows directly from the fact that $D_{0} / \mathfrak{A}$ is of rank $\nu$ over $K$, in view of our considerations on the congruences (8.1) and (8.2). Moreover, if we put $\lambda_{i}=\lambda_{i}^{*} / \xi_{0}^{* r_{i}}$, then $\lambda_{1}, \lambda_{2}, \cdots, \lambda_{\nu}$ are linearly independent modulo $\mathfrak{A}$, for if $\lambda_{1}, \lambda_{2}, \cdots, \lambda_{i-1}$ are independent $\bmod \mathfrak{A}$ while $\lambda_{i}=c_{1} \lambda_{1}+\cdots+c_{i-1} \lambda_{i-1}$ $+a \xi_{1}+b \xi_{2}$, then for a sufficiently large value of $k$ we have $\xi_{0}^{* k-r_{i}} \lambda_{i}^{*} \equiv c_{1} \xi_{0}^{* k-r_{1}} \lambda_{1}^{*}$ $+\cdots+c_{i-1} \xi_{0}^{* k-r_{i-1}} \lambda_{i-1}^{*}\left(\mathfrak{A}^{*}\right)$. The choice of $\lambda_{i}^{*}$ excludes the possibility of such a congruence. In a similar manner. we deduce that if $\omega^{*}$ is an element of $0^{*}$ which is homogeneous of degree $h$, and if, say, $r_{i}$ is the last element of the sequence $r_{1} \leqq r_{2} \leqq \cdots \leqq r_{\nu}$ such that $h \geqq r_{i}$ then $\omega^{*}$ satisfies a congruence of the form

$$
\xi_{0}^{* k-h} \omega^{*} \equiv \sum_{j=1}^{i} c_{j} \xi_{0}^{* k-r_{j}} \lambda_{j}^{*}\left(\mathfrak{A}^{*}\right)
$$

where $k$ is an integer. Since $h \geqq r_{j}, j=1,2, \cdots, i$, we can write

$$
\xi_{0}^{* k-h}\left(\omega^{*}-\sum_{j=1}^{i} c_{j} \xi_{0}^{* h-r_{j} \lambda}{ }_{j}^{*}\right) \equiv 0\left(\mathfrak{U}^{*}\right),
$$

and since $\mathfrak{A}^{*}$ has no projectively irrelevant components we have

$$
\omega^{*}-\sum_{j=1}^{i} c_{j} \xi_{0}^{* h-r_{j}} \lambda_{j}^{*}=A \xi_{1}^{*}+B \xi_{2}^{*}
$$

where $A$ and $B$ are in o* $^{*}$

We can now easily prove the following theorem.

THEOREM 8.2. The elements $\lambda_{1}^{*}, \lambda_{2}{ }^{*}, \cdots, \lambda_{\nu}^{*}$ form a modular base for $0^{*}$ over 
the ring $R^{*}$. In fact, if $\omega^{*}$ is a homogeneous element of degree $h$ in $0^{*}$, and $r_{i}$ is the last integer $r_{j}$ such that $h \geqq r_{j}$, then $\omega^{*}=P_{1} \lambda_{1}^{*}+\cdots+P_{i} \lambda_{i}^{*}$ where $P_{j}$ is in $R^{*}$ and is homogeneous of degree $h-r_{j}$.

Proof. If $h=0$ the assertion is trivially true. Assume, therefore, that the theorem is true for elements in $D^{*}$ which are homogeneous of degree less than $h$, and let $\omega^{*}$ be of degree $h$. We note that the left-hand side of (8.5) is homogeneous of degree $h$, so that we may assume that the elements $A$ and $B$ on the right-hand side of (8.5) are homogeneous of degree $h-1$. (This follows by the usual application of the mapping $\tau: \xi_{0}^{*} \rightarrow t \xi_{0}^{*}, t \in K$.) By induction, then, we have $A=\sum_{j=1}^{i} A_{j} \lambda_{j}^{*}, B=\sum_{j=1}^{i} B_{j} \lambda_{j}^{*}$, where $A_{j}$ and $B_{j}$ are homogeneous of degree $h-r_{j}-1, j=1, \cdots, i$. Hence by (8.5), $\omega^{*}=\sum P_{j} \lambda_{j}^{*}$ where

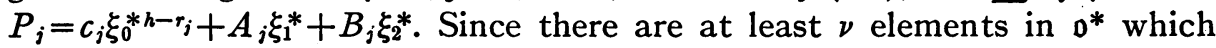
are linearly independent over $R^{*}$, the elements $\lambda_{1}^{*}, \lambda_{2}^{*}, \cdots, \lambda_{\nu}^{*}$ are necessarily independent over $R^{*}$, q.e.d.

\section{REFERENCES}

1. G. Castelnuovo, Alcune proprieta fondamentali dei sistemi lineari di curve tracciate sopra una superficie algebraica, Memorie di matematica e di fisica della Società Italiana delle Scienze (3) vol. 10 (1896).

2. R. Dedekind and H. Weber, Theorie der algebraischen Funktionen einer Veränderlichen, J. Reine Angew. Math. vol. 92 (1882).

3. W. Krull, Allgemeine Bewertungstheorie, J. Reine Angew. Math. vol. 167 (1932).

4. H. T. Muhly, A remark on normal varieties, Ann. of Math. vol. 42 (1941).

5. W. Schmeidler, Grundlagen einer Theorie der algebraischen Funktionen mehrerer Veränderlichen, Math. Zeit. vol. 28 (1928).

6. F. Severi, Sulla deficienza della serie caratteristica di un sistema lineare di curve appartenenti ad una superficie algebrica, Atti della Reale Accademia dei Lincei, Rendiconti (5) vol. 12 (1903).

7. B. L. van der Waerden, On Hilbert's function, series of composition of ideals, and a generalization of a theorem of Bezout, Proceedings of the Koninklijke Akademie van wetenschappen, Amsterdam vol. 31 (1928). (1935).

8. O. Zariski, Algebraic surfaces, Ergebnisse der Mathematik und ihrer Grenzgebiete vol. 3 (1939).

9. - Some results in the arithmetic theory of algebraic varieties, Amer. J. Math. vol. 61 (1940).

10. - Algebraic varieties over ground fields of characteristic zero, Amer. J. Math. vol. 62

11. - Pencils on an algebraic variety and a new proof of a theorem of Bertini, Trans. Amer. Math. Soc. vol. 50 (1941). (1942).

12. - Normal varieties and birational correspondences, Bull. Amer. Math. Soc. vol. 48

Princeton University,

Princeton, N. J.

U. S. Naval Academy,

Annapolis, MD. 